\title{
An Average Allowable Transmission Interval Condi- tion for the Stability of Networked Control Systems
}

\author{
Stefan H.J. Heijmans, Romain Postoyan, Dragan Nešić, Fellow, IEEE and W.P. Maurice H. Heemels, Fellow, IEEE
}

\begin{abstract}
A popular design framework for networked control systems (NCSs) is the emulation-based approach combined with Lyapunov-based analysis techniques for hybrid systems. In virtually all papers that use this framework, bounds in terms of the maximal allowable transmission interval (MATI) are provided to guarantee stability and performance properties of the NCS. However, having only such a MATI condition is rather restrictive and unrealistic in practice due to various network effects such as packet losses, leading to conservative bounds. In this paper, we therefore consider an alternative condition on the communication instants to better capture the time-varying properties of the transmission intervals. In particular, we propose, in addition to the existence of a MATI, to also impose a bound on the average allowable transmission interval, expressed in terms of a reverse average dwell-time (RADT) condition on the transmission intervals. We demonstrate by means of a novel Lyapunov-based analysis that stability of the NCS can still be guaranteed under this different condition on the transmission intervals, which can, in addition, lead to a significant improvement of the MATI. The strengths of these new results will be illustrated on a numerical example.
\end{abstract}

Index Terms-Networked control systems, stability of hybrid systems, Lyapunov methods, dwell-time conditions

\section{INTRODUCTION}

$\mathbf{N}$ ETWORKED control systems (NCSs) are systems in which the sensors, controllers, and/or actuators of the plant are physically distributed and communicate via (packetbased) digital channels. These systems have received considerable attention in recent years, motivated by the many benefits they offer with respect to conventional control systems, including greater flexibility, ease of maintenance, and low cost, weight and volume [1]-[6]. However, exploiting packet-based communication also comes with inevitable imperfections such as varying transmission intervals due to, for instance, packet losses, varying delays, and communication constraints that can all degrade the overall performance of the system and even lead to instability, see, e.g., [7] for an illustrative example. Moreover, as the communication network is often shared by multiple sensor and actuator nodes, there is a need for socalled scheduling protocols that govern the access of these nodes to the network.

To deal with these network-induced phenomena, several frameworks were developed in recent years to determine

S.H.J. Heijmans is with Demcon Advanced Mechatronics B.V. and W.P.M.H. Heemels is with the Department of Mechanical Engineering, Eindhoven University of Technology, The Netherlands. Their work is supported by the Innovational Research Incentives Scheme under the VICI grant 'Wireless control systems: A new frontier in automation' (No. 11382), which is financed by the Netherlands Organization for Scientific Research (NWO) R. Postoyan is with the Université de Lorraine, CNRS, CRAN, F-54000 Nancy, France. His work is supported by HANDY project ANR-18-CE400010-02. D. Nešić is with MIDAS Laboratory, the Department of Electrical and Electronic Engineering, the University of Melbourne, Australia. His work was supported under the Australian Research Council under the Discovery Project DP1094326.

E-mail corresponding author: s.h.j.heijmans@gmail.nl. conditions on the network while still guaranteeing stability and performance properties. A popular two-step design approach herein is the so-called emulation-based method as advocated in [8] and [9] combined with Lyapunov-based analysis tools for hybrid systems, reflected in the works [10]-[23]. The idea is to first design the (stabilizing) controller for the plant while ignoring the communication constraints. Note that in this first step any (nonlinear) controller design method can be used. In the second step, conditions on the communication rate, e.g., formalized through bounds on the transmission intervals using properties of the plant, controller, and scheduling protocol, are provided to guarantee closed-loop stability and performance of the overall NCS. In fact, a considerable amount of research has been focusing on determining the so-called maximal allowable transmission interval (MATI) for which the closed-loop NCS with the emulated controller is still stable, see, e.g., [8]-[23]. Hence, the problem of characterizing the MATI is instrumental in the analysis of NCSs.

However, a clear limitation of an analysis based only on a MATI condition as in, e.g., [8]-[23], is that the situation in which the length of each transmission interval is equal to this 'worst case' value (being the MATI bound) is allowed to occur and, therefore, needs to be covered in the analysis. Consequently, as this 'worst case' scenario is typically unrealistic to occur in practice since we have in general varying transmission intervals due to various network effects and large transmission intervals close to the MATI bound are rare, analyses from literature often lead to conservative bounds on the MATI. Indeed, it is, for instance, more likely to expect that situations occur at which the length of some transmission intervals is significantly larger than that of most transmission intervals (perhaps to cope with packet losses, which is particularly likely for wireless networks), provided that these 'longer' transmission intervals are somehow compensated for by a number of shorter inter-transmission times to maintain stability of the NCS. Such a scenario is, for instance, motivated by task executions on general purpose multiprocessor platforms that exhibit variations in their execution times, see [24]. As this feature cannot be addressed by only using the concept of a MATI, there is a need for a greater modeling flexibility to include more specific information on the transmission intervals in the NCS model.

We therefore propose in this work an alternative condition on the transmission intervals that better suits the above rationale and, thereby, connects better to the behavior of the communication networks in NCSs. That is, in addition to the existence of a MATI, we also propose to impose a bound on the average allowable transmission interval. In this way, stability of the NCS no longer only depends on the value for the (worst case) MATI, but also on the average value 
for the transmission intervals, allowing us to include more specific information regarding the possible varying length of the transmission intervals. Interestingly, this concept is directly related to exploiting a reverse average dwell-time (RADT) condition, as introduced in [25], [26] for hybrid systems, on the transmission instants, which translates to requiring that there is on average at least one transmission in the time interval of a length equal to the RADT. As such, we investigate in this work if imposing an additional RADT constraint on the transmission instants (cf. a bound on the average allowable transmission interval) leads to a significant improvement of the MATI for some transmission intervals with respect to the works of [12], [22], and [23], while maintaining the stability guarantees for the NCS.

In order to do so, we need to be able to not only integrate the MATI and the so-called minimal allowable transmission interval (MIATI), which always exists due to hardware limitations in terms of the smallest achievable sampling period for digital communication networks, but also the RADT into the hybrid model for NCSs as advocated in [11] and described in [12]. We therefore introduce in this work a new 'hybrid clock' to capture the MIATI, the MATI, and the RADT constraints in a hybrid model, allowing us to provide a new and extended hybrid model of the NCS itself. In addition, following [12], [23], we construct a novel, genuine (hybrid) Lyapunov function in the sense that it decreases during flows and does not increase during jumps of the hybrid system to show stability. Having such a genuine Lyapunov function is in contrast to earlier works on RADT conditions for hybrid systems as in [25], [26] since they used a Lyapunov function that is allowed to increase during flows, see also Remark 3 below. Moreover, the Lyapunov construction generalizes prior constructions from [12] and [22]. In summary, we provide for the first time a full Lyapunov-based proof for NCSs including a RADT condition. This novel result does, however, also introduce various new technical difficulties in the analysis. For instance, contrary to the previous works [10]-[12], [22] and [23], the newly introduced timer in the 'hybrid clock' to model the RADT constraint is no longer reset to zero at each transmission instant (see (6) below), which implies that the conditions under which the hybrid-timer dependent Lyapunov function decreases change significantly. This requires new analysis techniques as we will see below, among others, Lemma 1, which is instrumental in this aspect. Finally, the analysis shows that there exist certain trade-offs between the RADT and the MATI, which will also be evident in the considered numerical example. In particular, it will follow that when we transmit faster on average (i.e., a lower value for the RADT), we can obtain a significant improvement of the MATI. These novel results significantly extend our preliminary work [27] as, in this paper, we explicitly introduce the new 'hybrid clock' to capture the MIATI, MATI, and RADT constraints and provide full derivations and proofs. In addition, we show in this work how a bound on the maximal attainable improvement of the MATI can be computed a priori, which provides a direct indication of the improvements we can attain using these novel results and a detailed link to the so-called sampled-data (SD) case, see Section IV-E below.
The remainder of this paper is organized as follows. After presenting the necessary preliminaries and definitions in Section II, the considered class of systems is described in Section III, including a suitable hybrid model for the NCS. Here we also introduce the RADT condition and the new hybrid clock to include it in the hybrid model. In Section IV, we provide the sufficient Lyapunov conditions to guarantee uniformly globally exponential stability (UGES) of the NCS, after which we show how they can be applied to obtain a bound on the RADT and on the MATI (depending on the RADT). Here we also state our main result and give a detailed intuitive interpretation of the RADT constraint in the sampled-data (SD) case. Finally, in Section $\mathrm{V}$ we illustrate our results by means of a numerical example, showing that the MATI can indeed be significantly increased when having an additional RADT constraint, and in Section VI some concluding remarks are given.

\section{PRELIMINARIES}

The sets of real and natural numbers are denoted by $\mathbb{R}$ and $\mathbb{N}$, respectively, and the sets of non-negative real numbers and integers by $\mathbb{R}_{\geq 0}$ and $\mathbb{N}_{0}(:=\mathbb{N} \cup\{0\})$, respectively. For vectors $v_{1}, v_{2}, \ldots, v_{n} \in \mathbb{R}^{n}$, we denote by $\left(v_{1}, v_{2}, \ldots, v_{n}\right)$ the vector $\left[\begin{array}{llll}v_{1}^{\top} & v_{2}^{\top} & \cdots & v_{n}^{\top}\end{array}\right]^{\top}$, and by $|\cdot|$ and $\langle\cdot, \cdot\rangle$ the Euclidean norm and the usual inner product, respectively. Moreover, we define the distance of a vector $x \in \mathbb{R}^{n}$ to a closed set $A \subset \mathbb{R}^{n}$ as $|x|_{A}:=\min _{y \in A}|x-y|$. The $n$ by $n$ identity and zero matrices are denoted by $I_{n}$ and $0_{n}$, respectively. When the dimensions are clear from the context, these notations are simplified to $I$ and 0 .

Consider the nonlinear hybrid system given by

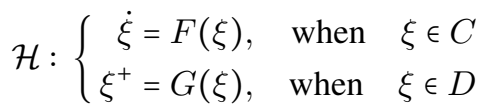

where $\xi \in \mathbb{X} \subseteq \mathbb{R}^{m_{\xi}}$ is the state vector, $F$ the flow map, $G$ the jump map, $C \subseteq \mathbb{X}$ the flow set, $D \subseteq \mathbb{X}$ is the jump set, and the set of initial conditions is defined as $\mathbb{X}_{0} \subseteq C \cup D \subseteq \mathbb{X}$ (i.e., $\left.\xi(0,0) \in \mathbb{X}_{0}\right)$. We assume that the sets $C$ and $D$ are closed sets and that $F$ and $G$ are continuous.

A solution to a hybrid system of the form (1) is defined on a hybrid time domain, which is a subset of $\mathbb{R}_{\geq 0} \times \mathbb{N}_{0}$. In particular, consider the following definitions from [28].

Definition 1a. A subset $E \subset \mathbb{R}_{\geq 0} \times \mathbb{N}_{0}$ is a compact hybrid time domain if $E=\bigcup_{j=0}^{J-1}\left(\left[t_{j}, t_{j+1}\right] \times\{j\}\right)$ for some finite sequence of times $0=t_{0} \leq t_{1} \leq t_{2} \leq \ldots \leq t_{J}$.

Definition 1b. A hybrid time domain is any set $E \subset \mathbb{R}_{\geq 0} \times \mathbb{N}_{0}$ such that for all $(T, J) \in E, E \cap([0, T] \times\{0,1, \ldots, J\})$ is a compact hybrid domain.

Definition 1c. A hybrid arc is a function $\xi$ defined on its hybrid time domain dom $\xi$ that is locally absolutely continuous in $t$ on the interval $(\operatorname{dom} \xi) \cap\left(\mathbb{R}_{\geq 0} \times\{j\}\right)$ for each $j \in \mathbb{N}_{0}$.

Definition 1d. A time instant $t \in \mathbb{R}_{\geq 0}$ is called a jump time for a hybrid arc $\xi$ if there is a $j \in \mathbb{N}$ such that $(t, j) \in \operatorname{dom} \xi$ and $(t, j-1) \in \operatorname{dom} \xi$.

We will often not mention dom $\xi$ explicitly, with the understanding that each hybrid arc $\xi$ is defined on its corresponding hybrid time domain $\operatorname{dom} \xi$. Moreover, observe that the sequence of times $0 \leq t_{1} \leq t_{2} \leq \ldots t_{J}$ as specified in Definition 
1a for the hybrid time domain in Definition $1 \mathrm{~b}$ (with $t_{0}$ thus excluded) are jump times. Using these definitions, similar to [28]-[30], we say now that a hybrid arc $\xi \in \mathbb{X}$ is a solution to (1) with initial state set $\mathbb{X}_{0}$ if $\xi(0,0) \in \mathbb{X}_{0}$ and

- for all $j \in \mathbb{N}_{0}$ and almost all $t$ such that $(t, j) \in \operatorname{dom} \xi$, $\xi(t, j) \in C$ and $\dot{\xi}(t, j)=F(\xi(t, j))$;

- for all $(t, j) \in \operatorname{dom} \xi$ such that $(t, j+1) \in \operatorname{dom} \xi, \xi(t, j) \in$ $D$ and $\xi(t, j+1)=G(\xi(t, j))$.

Hence, $\xi(t, j+1)$ is denoted $\xi^{+}$in (1) and $\operatorname{dom} \xi$ denotes the hybrid time domain of $\xi$. In addition, we say in this work that a solution $\xi$ is maximal, if there does not exist another solution $\tilde{\xi}$ such that $\operatorname{dom} \xi \subset \operatorname{dom} \tilde{\xi}$ and $\xi(t, j)=\tilde{\xi}(t, j)$ for all $(t, j) \in \operatorname{dom} \xi$, and that a solution $\xi$ is called $t$-complete if $\sup \left\{t \in \mathbb{R}_{\geq 0} \mid \exists j \in \mathbb{N}_{0}\right.$ such that $\left.(t, j) \in \operatorname{dom} \xi\right\}=\infty$. For a detailed description and interpretation of these concepts, the interested reader is referred to [28].

We are interested in the stability of the hybrid model (1).

Definition 2. Consider the hybrid system $\mathcal{H}$ on $\mathbb{X}$ given by (1) and consider a closed set $\mathcal{E} \subseteq \mathbb{X}_{0}$. Then the set $\mathcal{E}$ is said to be uniformly globally exponentially stable (UGES) if there exist constants $K, c>0$ such that for any initial condition $\xi(0,0) \in \mathbb{X}_{0}$, all corresponding maximal solutions $\xi$ are $t$ complete and satisfy for all $(t, j) \in \operatorname{dom} \xi$

$$
|\xi(t, j)|_{\mathcal{E}} \leq K|\xi(0,0)|_{\mathcal{E}} e^{-c(t+j)}
$$

\section{SySteM SETUP}

In this section, we introduce the NCS setup, the RADT condition, and a hybrid model describing the overall dynamics including a new hybrid clock, which captures the RADT constraint on the transmission instants.

\section{A. Networked Control Configuration}

We consider the NCS setup as shown in Fig. 1, where the continuous-time plant $\mathcal{P}$ communicates with the controller $\mathcal{C}$ via the network $\mathcal{N}$. We assume here that the controller is designed while ignoring the network, which is characteristic for the emulation-based design approach, as already mentioned in the introduction.

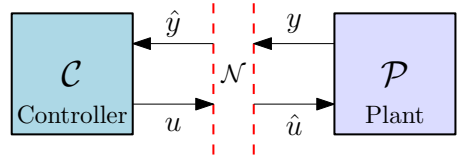

Fig. 1. The NCS setup as described in [8]-[23].

The plant and controller dynamics are given by

$$
\mathcal{P}:\left\{\begin{array}{c}
\dot{x}_{p}=f_{p}\left(x_{p}, \hat{u}\right) \\
y=g_{p}\left(x_{p}\right)
\end{array} \text { and } \mathcal{C}:\left\{\begin{array}{c}
\dot{x}_{c}=f_{c}\left(x_{c}, \hat{y}\right) \\
u=g_{c}\left(x_{c}\right),
\end{array}\right.\right.
$$

where $x_{p} \in \mathbb{R}^{m_{x_{p}}}$ and $x_{c} \in \mathbb{R}^{m_{x_{c}}}$ denote the plant and controller state, respectively, $u \in \mathbb{R}^{m_{u}}$ the control input, $\hat{u} \in \mathbb{R}^{m_{u}}$ the most recently received control input by the plant, $y \in \mathbb{R}^{m_{y}}$ the output, and $\hat{y} \in \mathbb{R}^{m_{y}}$ the most recently received output of the plant. We assume that $f_{p}$ and $f_{c}$ are continuous, and $g_{p}$ and $g_{c}$ continuously differentiable.

To complete the NCS setup, we also need to describe the network $\mathcal{N}$ in more detail. As such, we assume that it has a collection of sampling/transmission times $t_{j}, j \in \mathbb{N}$, which satisfy $0 \leq t_{1}<t_{2}<\ldots$. At such a time $t_{j}$, (parts of) the output $y$ and the input $u$ are sampled and transmitted to the controller $\mathcal{C}$ and the plant $\mathcal{P}$, respectively, which results in an update of (a part of) the networked values according to

$$
\begin{aligned}
& \hat{y}\left(t_{j}^{+}\right)=y\left(t_{j}\right)+h_{y}\left(j, e\left(t_{j}\right)\right) \\
& \hat{u}\left(t_{j}^{+}\right)=u\left(t_{j}\right)+h_{u}\left(j, e\left(t_{j}\right)\right),
\end{aligned}
$$

where the function $h:=\left(h_{y}, h_{u}\right)$ with $h: \mathbb{N}_{0} \times \mathbb{R}^{m_{e}} \rightarrow \mathbb{R}^{m_{e}}$ models the scheduling protocol that determines which (sensor and/or actuator) node is granted access to the network at time $t_{j}$, see, e.g., [11]-[15], and where $e$ denotes the networkinduced error defined by $e:=\left(e_{y}, e_{u}\right)=(\hat{y}-y, \hat{u}-u)$. We also assume that $\hat{y}$ and $\hat{u}$ are constant in between two successive transmissions (i.e., the network nodes operate in a similar manner to a zero-order-hold $(\mathrm{ZOH}))$. However, this can easily be modified, if desired, see [11].

Finally, as mentioned in the introduction and shown by the works of, e.g., [8]-[18], [20]-[23], it is common to assume that (all of) the transmission intervals are bounded by

$$
\tau_{\text {miati }} \leq t_{j+1}-t_{j} \leq \tau_{\text {mati }}, \quad j \in \mathbb{N},
$$

where $\tau_{m a t i}$ denotes the maximal allowable transmission interval (MATI) and $\tau_{\text {miati }}$ the minimal allowable transmission interval (MIATI), such that $0<\tau_{\text {miati }} \leq \tau_{\text {mati }}$. The upper bound $\tau_{\text {mati }}$ is used in many papers to guarantee stability properties of the NCS designed using the emulation-based approach, see, e.g., [12]-[23], while the lower bound on the transmission intervals $\tau_{\text {miati }}>0$ is often taken arbitrarily small, see, e.g., [8]-[21], since it is only imposed to prevent Zeno behavior. However, note that in practice this lower bound always exists due to limitations in digital communication hardware in terms of a smallest achievable transmission period. In fact, knowledge of the MIATI (instead of assuming it to be arbitrarily small) can be exploited to improve the MATI as was recently shown in [22] and [23] and as will also follow from our analysis (see Remark 7).

Combining all of the above, the NCS setup described by (2)(4) can be written in a hybrid system formalism as advocated in [11] and described in [12], which is of the form of (1) where each 'jump' of the hybrid system corresponds to an update of the networked values, i.e., the sequence of transmission times of the NCS is equal to the sequence of jump times of the hybrid model. Using this hybrid modeling framework, stability and performance of the NCS were analyzed in [12]-[16], [20], [22], [23] using a Lyapunov-based theorem for hybrid systems, resulting in values for the MATI such that, for instance, UGES for the NCS is guaranteed. Condition (4), however, allows for all of the transmission intervals to have a length equal to the MATI ('worst case'), which, as mentioned in the introduction, is often unrealistic in practice and conservative in the analysis. As such, our objective in this work is to include more specific information concerning the transmission intervals in the NCS model in terms of an average allowable transmission interval ('average case') to allow for significantly larger values for some specific transmission intervals equal to the MATI, while still ensuring stability. To do so, we rely on the concept of a RADT condition, which we will first introduce. 


\section{B. A Reverse Average Dwell-Time (RADT) Condition}

Introduced in [25] and [26] for a hybrid system given by (1), the RADT condition captures the situation in which continuous flow can potentially destroy the UGES property and must therefore be persistently interrupted by jumps of the hybrid system. In particular, it enforces a lower bound on the amount of jump times for any solution $\xi$ to (1) with its corresponding hybrid time domain ${ }^{1} \operatorname{dom} \xi$ by means of

$$
j-i \geq \frac{\bar{t}-t}{\tau_{r-d t}^{*}}-N_{0}, \quad \text { for all }(t, i),(\bar{t}, j) \in \operatorname{dom} \xi
$$

for some $N_{0} \geq 1$ and the appropriately chosen constant $\tau_{r-d t}^{*}>$ 0 called the RADT (see Section IV). Here, the quantity $j-i$ is, loosely speaking, related to the number of 'jumps' of the solution to the hybrid system between $t$ and $\bar{t}$.

The RADT condition (5) imposes that, on average, at least one jump of the hybrid system (1) occurs in an interval of length $\tau_{r-d t}^{*}$ time units and that for a fixed value of $N_{0} \geq 1$ there exists at most $N_{0} \tau_{r-d t}^{*}$ time units between two consecutive jumps, see [25], [26]. Moreover, in the special case of $N_{0}=1$, (5) actually recovers MATI-like bound as imposed by (the right hand-side of) the 'classical' condition (4), i.e., in the case of $N_{0}=1$, the RADT condition (5) requires that two consecutive transmission times must be separated by at most $\tau_{r-d t}^{*}$ time units, see also Remark 1 below. Hence, with imposing (5) on the jump (cf. transmission) times we do not only have an assumption on the RADT, but also on the MATI. In other words, having an analysis based on the concept of a RADT via (5) rather than having (only) a MATI bound for all transmission intervals as in condition (4) allows us to include more information regarding the varying lengths of the transmission intervals in the NCS model and forthcoming analysis. It is this extra condition on the RADT that will allow us to obtain higher bounds for the MATI than what can be obtained based on the analysis in [12] or [22]. We will therefore embed the RADT condition (5) (in addition to the, due to hardware limitations, always present, MIATI bound) in our NCS setup instead of using (4). To this end, we first introduce a 'hybrid clock' that allows us to include the RADT and the MIATI/MATI bounds in the hybrid model for the NCS as described in [10]-[12].

Remark 1. Observe that the RADT constraint of (5) thus also directly provides a bound on the maximal allowed time in between jumps of the hybrid system (cf. the MATI) in the form of the value $N_{0} \tau_{r-d t}^{*}$ for some $N_{0} \geq 1$. Indeed, in the case of NCSs we can take $N_{0} \geq \frac{\tau_{m a t i}}{\tau_{r_{-} d t}^{*}}$ with $\tau_{r-d t}^{*} \leq \tau_{m a t i}$ such that the RADT constraint (5) guarantees that there exists an upper bound smaller or equal to $\tau_{\text {mati }}$ on the transmission intervals $t_{j+1}-t_{j}, j \in \mathbb{N}$, of the NCS, similar to condition (4). Moreover, in the case of $N_{0}=1, \tau_{r-d t}^{*}$ actually represents a MATI for the NCS in the sense of condition (4), i.e., all transmission intervals may be at most of length $\tau_{m a t i}=\tau_{r-d t}^{*}$ time units. Note now that in [25], [26] the presence of such an upper bound $N_{0} \tau_{r-d t}^{*}$ on the transmission intervals is not exploited in the analysis as their stability results are independent of

\footnotetext{
${ }^{1}$ For each hybrid time domain there exists a natural (lexicographical) way of ordering the hybrid times, meaning that the condition $\bar{t}+j \geq t+i$ in (5) implies that either $\bar{t}>t$ or $t=\bar{t}$ and $j \geq i$, see [28, Section 2.2].
}

$N_{0} \geq 1$. Here, however, we do need the explicit upper bound $\tau_{m a t i} \leq N_{0} \tau_{r-d t}^{*}$ for the Lyapunov function construction, as we will see in Section IV below. Obviously, (5) with $N_{0} \geq \frac{\tau_{\text {mati }}}{\tau^{*}}$ also relates the values for $\tau_{r-d t}^{*}$ and $\tau_{m a t i}$ to each other, i.e., when we on average transmit slightly faster/slower (take $\tau_{r-d t}^{*}$ lower/higher), then $\tau_{m a t i}$ can be larger/smaller (as $N_{0}$ is allowed to be larger/smaller).

\section{A RADT Augmented Hybrid Model for the NCS}

Unfortunately, the existing hybrid clocks from literature do not incorporate the MIATI, the MATI and the RADT constraint simultaneously on the transmission instants. Indeed, existing hybrid clocks for NCSs as in [11] and [12] only model the constraint (4), while the hybrid clocks as in [29, Remark 17], [30, Proposition 3.2], or [31, Proposition 1.2] only model the RADT constraint (5). Therefore, we introduce in this paper a novel hybrid clock $\mathcal{T}$, consisting of the timer variable $\tau \in \mathbb{R}_{\geq 0}$ and the memory variable $s \in \mathbb{R}$ initialized at a value $s(0,0) \leq \tau_{\text {mati }}-\tau_{\text {miati }}$, and which is given by the hybrid system

$$
\mathcal{T}:\left\{\begin{array}{rlrl}
\dot{\tau}=1 & & & \text { when } \tau \in\left[0, \tau_{\text {mati }}\right] \\
\dot{s}=0 & & \text { when } \\
\tau^{+}=\max \left\{0, \tau-\tau_{r-d t}^{*}\right\} \\
s^{+}=\max \left\{0, \tau-\tau_{r-d t}^{*}\right\}
\end{array}\right\} \quad \begin{array}{ll}
\tau \in\left[s+\tau_{\text {miat }}, \tau_{\text {mati }}\right]
\end{array}
$$

for $0<\tau_{\text {miati }} \leq \tau_{r-d t}^{*} \leq \tau_{\text {mati }}$. The variable $s$ is used to 'remember' to which value $\tau$ was reset after a jump has occurred, which is needed to incorporate the MIATI bound, and is therefore reset to the same value as $\tau$ at a jump of the hybrid system but kept constant in between jump times. Moreover, the value for $\tau-s$ keeps track of the time elapsed since the last jump of the hybrid system (cf. the last transmission instant of the NCS), which is in contrast to the existing hybrid clocks in the NCS literature, see, e.g., [11], [12], where the timer $\tau$ itself already provided a direct indication of the elapsed time. This is a result of the value for $\tau$ in this case not (always) being reset to zero after a jump (cf. transmission) has occurred, but rather to the value $\max \{0, \tau-$ $\left.\tau_{r-d t}^{*}\right\}$, while in [11], [12] the clock $\tau$ is always reset to zero.

Combining the above properties, we can state the following.

Proposition 1. Let $0<\tau_{\text {miati }} \leq \tau_{r-d t}^{*} \leq \tau_{\text {mati }}$ be given. A hybrid time domain $E$ with its sequence of jump times $\left\{t_{j}\right\}_{j \in \mathbb{N}}$, satisfies

$$
\begin{aligned}
& t_{j+1}-t_{j} \geq \tau_{\text {miati }} \text { for all } j \in \mathbb{N} \\
& j-i \geq \frac{(\bar{t}-t)-\tau_{\text {mati }}}{\tau_{r-d t}^{*}} \text { for all }(t, i),(\bar{t}, j) \in E \\
& \text { with } \bar{t}+j \geq t+i
\end{aligned}
$$

if and only if $E=\operatorname{dom}(\tau, s)$ for some solution $(\tau, s)$ to (6) with initial state set $\mathbb{X}_{0}=\left[0, \tau_{\text {mati }}\right] \times\left(-\infty, \tau_{\text {mati }}-\tau_{\text {miati }}\right]$.

The proof is given in Appendix A. Note that condition (7a) corresponds to ensuring that two consecutive jumps of the hybrid clock are separated by at least $\tau_{\text {miati }}$ time units, while condition (7b) is equivalent to (5) with $N_{0}=\frac{\tau_{m a t i}}{\tau_{r-d t}^{*}}$ (and therefore models the RADT and the MATI constraints, see 
Remark 1). Hence, this new hybrid clock takes care of the MIATI, the MATI, and the constraint on the average allowable transmission interval expressed by means of the RADT.

Combining now the continuous-time properties of the plant and the controller as given by (2), the network protocol (3), and the timing properties of the network modeled using the hybrid clock $\mathcal{T}$ in (6), the NCS setup can be expressed as a hybrid model of the form (1), where each jump of the hybrid system corresponds to an update of the networked values and that satisfies both (4) and (5) (cf. (7)). To do so, for the modeling of certain scheduling protocols, we also need to introduce a counter $\kappa \in \mathbb{N}_{0}$, which keeps track of the number of transmissions, see [11], [12]. In particular, following from (3), we consider the class of scheduling protocol functions $h: \mathbb{N}_{0} \times \mathbb{R}^{m_{e}} \rightarrow \mathbb{R}^{m_{e}}$ for which the error dynamics at jumps of the hybrid system are given by

$$
e^{+}=h(\kappa, e) \text {. }
$$

Examples of such scheduling protocols are, among others, the sampled-data (SD), round-robin (RR), and try-once-discard (TOD) protocols [11].

Based on the above and following [11] and [12], we thus obtain that the NCS described by (2)-(5) can be expressed as the hybrid system $\mathcal{H}_{n c s}$ given by

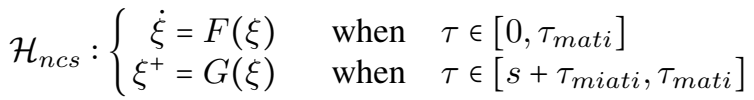

with its full state $\xi:=\left(\left(x_{p}, x_{c}\right), e,(\tau, s), \kappa\right) \in \mathbb{X}:=\mathbb{R}^{m_{x}} \times$ $\mathbb{R}^{m_{e}} \times\left[0, \tau_{\text {mati }}\right] \times \mathbb{R} \times \mathbb{N}_{0}$ and set of initial conditions $\mathbb{X}_{0}:=\mathbb{R}^{m_{x}} \times \mathbb{R}^{m_{e}} \times\left[0, \tau_{\text {mati }}\right] \times\left(-\infty, \tau_{\text {mati }}-\tau_{\text {miati }}\right] \times \mathbb{N}_{0}$, and where $F(\xi):=(f(x, e), g(x, e), 1,0,0)$ and $G(\xi):=$ $\left(x, h(\kappa, e), \max \left\{0, \tau-\tau_{r-d t}^{*}\right\}, \max \left\{0, \tau-\tau_{r-d t}^{*}\right\}, \kappa+1\right)$ with $x:=\left(x_{p}, x_{c}\right) \in \mathbb{R}^{m_{x}}, m_{x}=m_{x_{p}}+m_{x_{c}}$, and

$$
\begin{aligned}
& f(x, e)::=\left[\begin{array}{l}
f_{p}\left(x_{p}, g_{c}\left(x_{c}\right)+e_{u}\right) \\
f_{c}\left(x_{c}, g_{p}\left(x_{p}\right)+e_{y}\right)
\end{array}\right] \\
& g(x, e):=\left[\begin{array}{l}
-\frac{\partial g_{p}}{\partial x_{p}} f_{p}\left(x_{p}, g_{c}\left(x_{c}\right)+e_{u}\right) \\
-\frac{\partial g_{c}}{\partial x_{c}} f_{c}\left(x_{c}, g_{p}\left(x_{p}\right)+e_{y}\right)
\end{array}\right] .
\end{aligned}
$$

Using this augmented hybrid system (9), we will now analyze for which pairs of $\left(\tau_{r-d t}^{*}, \tau_{\text {mati }}\right)$ the set

$$
\mathcal{E}:=\{\xi \in \mathbb{X} \mid x=0 \text { and } e=0\}
$$

is guaranteed to be UGES for the NCS.

\section{A LyapunOV-BASEd Stability AnAlysis}

A fundamental tool in the stability analysis for hybrid systems (and in particular NCSs) is the use of Lyapunov theorems and its corresponding Lyapunov function [12], [28]. Therefore, in this section, we analyze the stability of the hybrid model (9) for the NCS by means of constructing a genuine Lyapunov function in the sense that it strictly decreases during flows (i.e., in between transmission times) and does not increase during jumps (i.e., when an update of the networked values occurs), see also (11) below. This will directly lead to conditions on the RADT and the MATI such that UGES of the set $\mathcal{E}$ is guaranteed.

\section{A. The Lyapunov Function and Conditions}

Following [12], [28] and in line with the works [13]-[15], [21] and [22], we say that a function $U: \mathbb{X} \rightarrow \mathbb{R}_{\geq 0}$ is a (hybrid) Lyapunov function for the hybrid system (9) if $U$ is locally Lipschitz, positive definite, and radially unbounded with respect to the set $\mathcal{E}$ in the sense that there exist constants $\underline{\alpha}_{U}, \bar{\alpha}_{U}>0$ such that for all $\xi \in \mathbb{X}$

$$
\underline{\alpha}_{U}|\xi|_{\mathcal{E}}^{2} \leq U(\xi) \leq \bar{\alpha}_{U}|\xi|_{\mathcal{E}}^{2}
$$

and if $U$ satisfies for some constant $\delta>0$ for (almost) all $\xi \in \mathbb{X}$

$$
\begin{gathered}
\langle\nabla U(\xi), F(\xi)\rangle \leq-\delta U(\xi), \text { when } \tau \in\left[0, \tau_{\text {mati }}\right] \\
U(G(\xi))-U(\xi) \leq 0, \text { when } \tau \in\left[s+\tau_{\text {miati }}, \tau_{\text {mati }}\right]
\end{gathered}
$$

with $F(\xi)$ and $G(\xi)$ as in (9). Based on (11) and the fact that there is always at least $\tau_{\text {miati }}>0$ time units between jumps (as a result of Proposition 1), we have the following result, commonly known in the hybrid literature.

Theorem 1. Consider the hybrid system $\mathcal{H}_{n c s}$ on $\mathbb{X}$ given by (9). If there exists a Lyapunov function $U: \mathbb{X} \rightarrow \mathbb{R}_{\geq 0}$ for $\mathcal{H}_{n c s}$, then the set $\mathcal{E}$ given by (10) is UGES.

The proof directly follows from the proof of [12, Theorem 1]. To obtain now bounds on the values for the RADT and the MATI (which will depend on the value for the RADT as we will see below) for which the NCS is UGES, we aim to construct a Lyapunov function $U$ for the hybrid system (9), which satisfies (11). To do so, in line with [11]-[13], we first make the following assumption.

Assumption 1. Consider the hybrid system $\mathcal{H}_{n c s}$ as in (9). There exist a function $W: \mathbb{N}_{0} \times \mathbb{R}^{m_{e}} \rightarrow \mathbb{R}_{\geq 0}$ that is locally Lipschitz in its second argument, a locally Lipschitz function $V: \mathbb{R}^{m_{x}} \rightarrow \mathbb{R}_{\geq 0}$, a continuous function $H: \mathbb{R}^{m_{x}} \rightarrow \mathbb{R}$, and constants $\lambda \in(0,1), L \geq 0, \underline{\alpha}_{W}, \bar{\alpha}_{W}, \underline{\alpha}_{V}, \bar{\alpha}_{V}, \varepsilon, \gamma>0$ such that the following hold:

1) For all $\kappa \in \mathbb{N}_{0}$ and $e \in \mathbb{R}^{m_{e}}$

$$
\begin{aligned}
\underline{\alpha}_{W}|e| \leq W(\kappa, e) & \leq \bar{\alpha}_{W}|e| \\
W(\kappa+1, h(\kappa, e)) & \leq \lambda W(\kappa, e) .
\end{aligned}
$$

2) For all $\kappa \in \mathbb{N}_{0}, x \in \mathbb{R}^{m_{x}}$, and almost all $e \in \mathbb{R}^{m_{e}}$

$$
\left|\frac{\partial W(\kappa, e)}{\partial e}, g(x, e)\right| \leq L W(\kappa, e)+H(x) .
$$

3) For all $x \in \mathbb{R}^{m_{x}}$

$$
\underline{\alpha}_{V}|x|^{2} \leq V(x) \leq \bar{\alpha}_{V}|x|^{2} .
$$

4) For all $\kappa \in \mathbb{N}_{0}, e \in \mathbb{R}^{m_{e}}$, and almost all $x \in \mathbb{R}^{m_{x}}$

$$
\langle\nabla V(x), f(x, e)\rangle \leq-\varepsilon^{2} V(x)-H^{2}(x)+\gamma^{2} W^{2}(\kappa, e) .
$$

This assumption is essentially the same as the main assumption [12, Assumption 1]. Moreover, these conditions (12)-(15) have been proven to be systematically checkable for various classes of NCSs, see, e.g., [15]-[22]. In particular, we can divide the assumption in a part that is related to stability of the error dynamics of the NCS and a part that is related to stability of the closed-loop dynamics of the NCS, which is characteristic for the emulation-based approach for NCSs 
as exploited in this paper. To be more precise, condition (12) means that the scheduling protocol is UGES, a notion introduced in [11], and is therefore directly related to the 'discrete' error dynamics (8) of the NCS. The SD, RR, and TOD protocols are, among others, UGES scheduling protocols, for which the functions $W$ are provided in [11]. On the other hand, conditions (14) and (15) are related to finding a Lyapunov function for the closed-loop dynamics $\dot{x}=f(x, e)$ that establishes an $\mathcal{L}_{2}$-gain $\gamma$ from $W$ to $H$. The remaining part is then to connect condition (12) on the one hand and conditions (14) and (15) on the other hand, which is done via condition (13), which is an exponential growth condition on $W$ along the solutions of the error system $\dot{e}=g(x, e)$ between two consecutive transmission instants. For more information concerning this type of analysis we refer to [11], [12].

In view of Assumption 1 and inspired by [12], [22], we propose for the hybrid system (9) to take, for any $\xi \in \mathbb{X}$,

$$
U(\xi)=\phi_{V}(\tau) V(x)+\gamma \phi_{W}(\tau) W^{2}(\kappa, e)
$$

as a candidate Lyapunov function where $\phi_{V}, \phi_{W}:\left[0, \tau_{\text {mati }}\right] \rightarrow$ $\mathbb{R}_{\geq 0}$ are some functions to be designed. Certainly, these functions need to have strictly positive lower and upper bounds, i.e., there exist constants $\underline{\phi}_{V}^{c}, \bar{\phi}_{V}^{c}, \underline{\phi}_{W}^{c}, \bar{\phi}_{W}^{c} \in \mathbb{R}_{>0}$ such that

$$
\begin{gathered}
\phi_{V}^{c} \leq \phi_{V}(\tau) \leq \bar{\phi}_{V}^{c} \\
\underline{\phi}_{W}^{c} \leq \phi_{W}(\tau) \leq \bar{\phi}_{W}^{c}
\end{gathered}
$$

for all $\tau \in\left[0, \tau_{m a t i}\right]$. Note that (17) in combination with (12a) and (14) will guarantee that $U$ satisfies (11a).

The objective is now to construct the functions $\phi_{V}$ and $\phi_{W}$ to ensure (11b)-(11c) and (17). A reader, who is not interested in the proof techniques, may directly go to Section IV-D to find the statement of the main result.

Remark 2. The construction of the Lyapunov function as given by (16) generalizes prior constructions from [12], [22] for NCSs by means of the functions $\phi_{V}$ and $\phi_{W}$, see also [23]. Indeed, by choosing $\phi_{V}(\tau)=1$ for all $\tau \in \mathbb{R}_{\geq 0}$ and $\phi_{W}$ as in [12, Claim 1], we recover the Lyapunov function as used in [12], [22] as a special case. It is this flexibility with respect to $\phi_{V}$ and $\phi_{W}$ that allows us to exploit the RADT condition in a Lyapunov-based analysis for NCSs, as we will see below.

Remark 3. The Lyapunov conditions as in (11) can be directly linked to the 'non-monotonic' Lyapunov conditions ${ }^{2}$ that are typically used for verifying stability of hybrid systems that exploit a RADT condition like (5), as stated in, e.g., [25], [26]. That is, based on the result from [26, Corollary 1], it follows that UGES for the hybrid system $\mathcal{H}_{n c s}$ given by (9) is guaranteed when, for some $\tau_{r-d t}^{*}<\frac{d}{|c|}$ with $d>0$ and $c<0$, there exists a 'non-monotonic' Lyapunov function $\mathcal{U}$ satisfying for some constants $\underline{\alpha}_{\mathcal{U}}, \bar{\alpha}_{\mathcal{U}}>0$ for (almost) all $\xi \in \mathbb{X}$

$$
\begin{aligned}
& \underline{\alpha}_{\mathcal{U}}|\xi|_{\mathcal{E}}^{2} \leq \mathcal{U}(\xi) \leq \bar{\alpha}_{\mathcal{U}}|\xi|_{\mathcal{E}}^{2} \\
& \langle\nabla \mathcal{U}(\xi), F(\xi)\rangle \leq-c \mathcal{U}(\xi), \text { when } \tau \in\left[0, \tau_{\text {mati }}\right] \\
& \mathcal{U}(G(\xi)) \leq e^{-d} \mathcal{U}(\xi), \text { when } \tau \in\left[s+\tau_{\text {miati }}, \tau_{\text {mati }}\right] .
\end{aligned}
$$

\footnotetext{
${ }^{2}$ A Lyapunov function is called 'non-monotonic' when it is allowed to increase along the continuous or discrete dynamics, see [32]-[34].
}

The Lyapunov conditions of (18) translate to the 'nonmonotonic' Lyapunov function $\mathcal{U}$ being allowed to increase during flows of the hybrid system $\mathcal{H}_{n c s}$, but decreases during jumps. As such, the Lyapunov conditions (18) imply that, when there is a sufficient amount of jumps (i.e., updates of the networked values) expressed by means of a bound on $\tau_{r-d t}^{*}$, the overall 'non-monotonic' Lyapunov function $\mathcal{U}$ will decrease over time and stability of the NCS is guaranteed, which is consistent with the concept of RADT as discussed in Section III-B. Indeed, from $\mathcal{U}$ with $\tau_{r-d t}^{*}<\frac{d}{|c|}$ we can directly construct a genuine Lyapunov function $U$ satisfying (11). In particular, consider for some $\tilde{c}>|c|$ the function, $\xi \in \mathbb{X}$,

$$
U(\xi)=e^{-\tilde{c} \tau} \mathcal{U}(\xi)
$$

and recall that $\tau \in \mathbb{R}_{\geq 0}$ models part of the timer with $\dot{\tau}=1$ and $\tau^{+}=\max \left\{0, \tau-\tau_{r-d t}^{*}\right\}$. We observe that (18b) implies during flows, for almost all $\xi \in \mathbb{X}$ with $\tau \in\left[0, \tau_{\text {mati }}\right]$, that

$$
\begin{array}{r}
\langle\nabla U(\xi), F(\xi)\rangle=-\tilde{c} \dot{\tau} e^{-\tilde{c} \tau} \mathcal{U}(\xi)+e^{-\tilde{c} \tau}\langle\nabla \mathcal{U}(\xi), F(\xi)\rangle \\
\stackrel{(18 \mathrm{~b})}{\leq}(-\tilde{c}-c) e^{-\tilde{c} \tau} \mathcal{U}(\xi)=(-\tilde{c}-c) U
\end{array}
$$

and, hence, we indeed have that the function $U$ is strictly decreasing since $\tilde{c}>|c|$. Moreover, at a jump of the hybrid system $\mathcal{H}_{n c s},(18 \mathrm{c})$ implies that for $\xi \in \mathbb{X}$ with $\tau \in\left[s+\tau_{\text {miati }}, \tau_{\text {mati }}\right]$

$$
\begin{aligned}
U(G(\xi)) & =e^{-\tilde{c} \max \left\{0, \tau-\tau_{r-d t}^{*}\right\}} \mathcal{U}(G(\xi)) \\
& \stackrel{(18 c)}{\leq} e^{-\tilde{c} \max \left\{0, \tau-\tau_{r-d t}^{*}\right\}} e^{-d} \mathcal{U}(\xi) \\
& \stackrel{(19))}{=} e^{-\tilde{c} \max \left\{0, \tau-\tau_{r-d t}^{*}\right\}} e^{-d} e^{\tilde{c} \tau} U(\xi) \\
& =e^{\tilde{c} \min \left\{\tau, \tau_{r-d t}^{*}\right\}} e^{-d} U(\xi) .
\end{aligned}
$$

Hence, by observing now that, for $\tau_{r-d t}^{*} \leq \frac{d}{\tilde{c}}$, we have that

$$
\begin{aligned}
\min \left\{\tau, \tau_{r-d t}^{*}\right\} \leq \frac{d}{\tilde{c}} & \Rightarrow \tilde{c} \min \left\{\tau, \tau_{r-d t}^{*}\right\}-d \leq 0 \\
& \Rightarrow e^{\tilde{c} \min \left\{\tau, \tau_{r-d t}^{*}\right\}} e^{-d} \leq 1
\end{aligned}
$$

and that the value for $\tilde{c}$ can be chosen arbitrarily close to the value $|c|$, also (11c) is satisfied for $U$ since $\tau_{r-d t}^{*}<\frac{d}{|c|}$. As such, if the standard RADT-like 'non-monotonic' Lyapunov function construction based on (18) guarantees UGES for the NCS, we can also work with a genuine hybrid Lyapunov function satisfying (11). Moreover, since our model $\mathcal{H}_{n c s}$ already embeds the RADT constraint via the hybrid clock (6), it is more elegant to work with the Lyapunov conditions of (11) rather than (18) (otherwise we would have to invoke the RADT constraint twice as it is included in our model and needs to be used in combination with (18), which is unnatural in our view). Hence, to obtain a cleaner analysis and 'simpler' conditions on the RADT/MATI, we prefer to directly construct a Lyapunov function satisfying (11).

\section{B. Designing the Functions $\phi_{V}$ and $\phi_{W}$}

Firstly, consider condition (11b). We obtain for almost all $(x, e) \in \mathbb{R}^{m_{x}} \times \mathbb{R}^{m_{e}}$ and all $(\kappa, \tau) \in \mathbb{N}_{0} \times\left[0, \tau_{\text {mati }}\right]$ that

$$
\begin{aligned}
\langle\nabla U(\xi), F(\xi)\rangle & =\phi_{V}(\tau)\langle\nabla V(x), f(x, e)\rangle \\
+ & \gamma\left(\frac{\mathrm{d}}{\mathrm{d} \tau} \phi_{W}(\tau)\right) W^{2}(\kappa, e)+\left(\frac{\mathrm{d}}{\mathrm{d} \tau} \phi_{V}(\tau)\right) V(x) \\
+ & 2 \gamma \phi_{W}(\tau) W(\kappa, e)\left\langle\frac{\partial W(\kappa, e)}{\partial e}, g(x, e)\right\rangle,
\end{aligned}
$$


which implies that

$$
\begin{aligned}
\langle\nabla U(\xi), & F(\xi)\rangle \\
\stackrel{(13),(15)}{\leq} & \left(\frac{\mathrm{d}}{\mathrm{d} \tau} \phi_{V}(\tau)\right) V(x)+\gamma\left(\frac{\mathrm{d}}{\mathrm{d} \tau} \phi_{W}(\tau)\right) W^{2}(\kappa, e) \\
& +\phi_{V}(\tau)\left(-\varepsilon^{2} V(x)-H^{2}(x)+\gamma^{2} W^{2}(\kappa, e)\right) \\
& +2 \gamma \phi_{W}(\tau) W(\kappa, e)(L W(\kappa, e)+H(x)) \\
= & \left(\frac{\mathrm{d}}{\mathrm{d} \tau} \phi_{V}(\tau)-\varepsilon^{2} \phi_{V}(\tau)\right) V(x)-\phi_{V}(\tau) H^{2}(x) \\
& +\gamma^{2} \phi_{V}(\tau) W^{2}(\kappa, e)+\gamma\left(\frac{\mathrm{d}}{\mathrm{d} \tau} \phi_{W}(\tau)\right) W^{2}(\kappa, e) \\
& +2 \gamma \phi_{W}(\tau) L W^{2}(\kappa, e)+2 \gamma \phi_{W}(\tau) H(x) W(\kappa, e) .
\end{aligned}
$$

Although there are at this point multiple possibilities in choosing the functions $\phi_{V}$ and $\phi_{W}$, it follows from the above inequality that a natural choice for $\phi_{V}$ is to take it as the positive nondecreasing exponential function given for some small enough constant $\delta>0$ (i.e., $\delta \leq \varepsilon^{2}$ ) by

$$
\frac{\mathrm{d}}{\mathrm{d} \tau} \phi_{V}(\tau)=\left(\varepsilon^{2}-\delta\right) \phi_{V}(\tau) \Leftrightarrow \phi_{V}(\tau)=e^{\left(\varepsilon^{2}-\delta\right) \tau} \phi_{V}(0)
$$

with $\phi_{V}(0)=\underline{\phi}_{V}^{c}>0$, satisfying (17a). Moreover, when we observe that

$$
\begin{aligned}
2 \gamma \phi_{W}(\tau) & H(x) W(\kappa, e) \\
& =2 \gamma \phi_{W}(\tau) \sqrt{\phi_{V}(\tau)} \frac{1}{\sqrt{\phi_{V}(\tau)}} H(x) W(\kappa, e) \\
& \leq \gamma^{2} \frac{\phi_{W}^{2}(\tau)}{\phi_{V}(\tau)} W^{2}(\kappa, e)+\phi_{V}(\tau) H^{2}(x),
\end{aligned}
$$

we obtain, in combination with (20), that

$$
\begin{aligned}
& \langle\nabla U(\xi), F(\xi)\rangle \\
& \leq-\delta \phi_{V}(\tau) V(x)+\gamma\left(\frac{\mathrm{d}}{\mathrm{d} \tau} \phi_{W}(\tau)\right) W^{2}(\kappa, e) \\
& +\left(2 \gamma L \phi_{W}(\tau)+\gamma^{2}\left(\frac{\phi_{W}^{2}(\tau)}{\phi_{V}(\tau)}+\phi_{V}(\tau)\right)\right) W^{2}(\kappa, e) .
\end{aligned}
$$

As such, it also follows that a convenient choice for $\phi_{W}$ : $\left[0, \tau_{\text {mati }}\right] \rightarrow \mathbb{R}_{\geq 0}$ is to take it according to the (nonlinear) differential equation

$$
\begin{aligned}
\frac{\mathrm{d}}{\mathrm{d} \tau} \phi_{W}(\tau)= & -2 L \phi_{W}(\tau)-\gamma\left(\frac{\phi_{W}^{2}(\tau)}{\phi_{V}(\tau)}+\phi_{V}(\tau)\right) \\
& -\delta \phi_{W}(\tau),
\end{aligned}
$$

because, when choosing so, we obtain that

$$
\begin{aligned}
\langle\nabla U(\xi), F(\xi)\rangle & \leq-\delta \phi_{V}(\tau) V(x)-\delta \gamma \phi_{W}(\tau) W^{2}(\kappa, e) \\
& =-\delta U(\xi),
\end{aligned}
$$

which implies that $(11 b)$ is satisfied.

Remark 4. Note that the choice of (21) for the function $\phi_{W}$ follows from the analysis in a similar fashion as was the case in the analysis of [12, Theorem 1], although we obtain a different differential equation here.

It is interesting and important to observe that (21) is a socalled Chini equation [35], [36], which is of the general form

$$
\frac{\mathrm{d}}{\mathrm{d} \tau} y(\tau)=f(\tau) y^{n}(\tau)-g(\tau) y(\tau)+h(\tau)
$$

with $n=2, f(\tau)=-\frac{\gamma}{\phi_{V}(\tau)}, g(\tau)=2 L+\delta$, and $h(\tau)=$ $-\gamma \phi_{V}(\tau)$. Moreover, as the condition number, see [37, p. 303],

$$
\begin{gathered}
f(\tau)^{-n-1} h(\tau)^{-2 n+1}\left(f(\tau)\left(\frac{\mathrm{d}}{\mathrm{d} \tau} h(\tau)\right)-\left(\frac{\mathrm{d}}{\mathrm{d} \tau} f(\tau)\right) h(\tau)\right. \\
-n g(\tau) f(\tau) h(\tau))^{n} \\
=\frac{4}{\gamma^{2}}\left(2 L+2 \delta-\varepsilon^{2}\right)^{2}
\end{gathered}
$$

is independent of $\tau$, it follows that we can express the function $\phi_{W}$ by means of a separation of variables given by

$$
\phi_{W}(\tau)=\left(\frac{h(\tau)}{f(\tau)}\right)^{1 / n} \tilde{\phi}_{W}(\tau)=\phi_{V}(\tau) \tilde{\phi}_{W}(\tau)
$$

where the function $\tilde{\phi}_{W}:\left[0, \tau_{\text {mati }}\right] \rightarrow \mathbb{R}_{\geq 0}$ is given by the (nonlinear) differential equation

$$
\frac{\mathrm{d}}{\mathrm{d} \tau} \tilde{\phi}_{W}(\tau)=-\left(2 L+\varepsilon^{2}\right) \tilde{\phi}_{W}(\tau)-\gamma\left(\tilde{\phi}_{W}^{2}(\tau)+1\right),
$$

which is the same as the function $\phi$ in [12, Claim 1] for $\varepsilon=0$. Indeed, from (23) if follows that

$$
\begin{aligned}
\frac{\mathrm{d}}{\mathrm{d} \tau} \phi_{W}(\tau)= & \left(\frac{\mathrm{d}}{\mathrm{d} \tau} \phi_{V}(\tau)\right) \tilde{\phi}_{W}(\tau)+\phi_{V}(\tau)\left(\frac{\mathrm{d}}{\mathrm{d} \tau} \tilde{\phi}_{W}(\tau)\right) \\
= & +\phi_{V}(\tau)\left(-(2 L) \varepsilon^{2}-\delta\right) \phi_{V}(\tau) \tilde{\phi}_{W}(\tau) \\
= & \left(\varepsilon^{2}-\delta\right) \phi_{V}(\tau) \frac{\phi_{W}(\tau)}{\phi_{V}(\tau)} \\
& +\phi_{V}(\tau)\left(-\left(2 L+\varepsilon^{2}\right) \frac{\phi_{W}(\tau)}{\phi_{V}(\tau)}-\gamma\left(\frac{\phi_{W}^{2}(\tau)}{\phi_{V}^{2}(\tau)}+1\right)\right) \\
= & -(2 L+\delta) \phi_{W}(\tau)-\gamma\left(\frac{\phi_{W}^{2}(\tau)}{\phi_{V}(\tau)}+\phi_{V}(\tau)\right),
\end{aligned}
$$

which is indeed (21). Hence, for the Lyapunov function $U$ of (16) with the choices of $\phi_{V}$ and $\phi_{W}$ as given by (20) and (21), respectively, we actually have that

$$
U(\xi)=\phi_{V}(\tau)\left(V(x)+\gamma \tilde{\phi}_{W}(\tau) W^{2}(\kappa, e)\right) .
$$

Using this result, we now shift our attention to condition (11c) by considering the situation in which an update of the networked values is performed, i.e., a jump occurs in the hybrid system (9) with $\tau \in\left[s+\tau_{\text {miati }}, \tau_{\text {mati }}\right]$. This gives for $\xi \in \mathbb{X}$

$$
\begin{aligned}
U(G(\xi)) & =\phi_{V}\left(\max \left\{0, \tau-\tau_{r-d t}^{*}\right\}\right)(V(x) \\
& \left.+\gamma \tilde{\phi}_{W}\left(\max \left\{0, \tau-\tau_{r-d t}^{*}\right\}\right) W^{2}(\kappa+1, h(\kappa, e))\right) \\
\leq & e^{-\left(\varepsilon^{2}-\delta\right) \tau_{m i a t i}} \phi_{V}(\tau)(V(x) \\
& \left.+\gamma \tilde{\phi}_{W}\left(\max \left\{0, \tau-\tau_{r-d t}^{*}\right\}\right) \lambda^{2} W^{2}(\kappa, e)\right) .
\end{aligned}
$$

The first term in (26), i.e., $e^{-\left(\varepsilon^{2}-\delta\right) \tau_{\text {miati }}} \phi_{V}(\tau)$, follows from the fact that $\phi_{V}$ is a nondecreasing function according to (20) and, as such, it decreases in value when $\tau$ decreases in value (which is the case during a jump of the system). In particular, for any $\tau_{1}, \tau_{2} \in \mathbb{R}_{\geq 0}$, we have that

$$
\phi_{V}\left(\tau_{2}\right)=e^{\left(\varepsilon^{2}-\delta\right)\left(\tau_{2}-\tau_{1}\right)} \phi_{V}\left(\tau_{1}\right)
$$


As such, when $\tau_{1}=\tau$ and $\tau_{2}=\max \left\{0, \tau-\tau_{r-d t}^{*}\right\}$ (and, hence, $\tau_{1}>\tau_{2}$ ), we obtain that

$$
\begin{aligned}
\phi_{V}\left(\max \left\{0, \tau-\tau_{r-d t}^{*}\right\}\right) & =e^{\left(\varepsilon^{2}-\delta\right) \max \left\{-\tau,-\tau_{r-d t}^{*}\right\}} \phi_{V}(\tau) \\
& =e^{-\left(\varepsilon^{2}-\delta\right) \min \left\{\tau, \tau_{r-d t}^{*}\right\}} \phi_{V}(\tau) \\
& \leq e^{-\left(\varepsilon^{2}-\delta\right) \tau_{\text {miati }}} \phi_{V}(\tau)
\end{aligned}
$$

since $\tau \geq \tau_{\text {miati }}$ (due to the fact that a jump of the system can only take place when $\tau \geq s+\tau_{\text {miati }}$ with $s \geq 0$, see also Proposition 1) and $\tau_{\text {miati }} \leq \tau_{r-d t}^{*}$. Observe now that

$$
e^{-\left(\varepsilon^{2}-\delta\right) \tau_{\text {miati }}} \phi_{V}(\tau) \leq \phi_{V}(\tau)
$$

since $\delta \leq \varepsilon^{2}$, which is required for (11c) to be satisfied.

For the second term in (26), it follows that, in order for (11c) to hold, we should also have that

$$
e^{-\left(\varepsilon^{2}-\delta\right) \tau_{\text {miati }}} \lambda^{2} \tilde{\phi}_{W}\left(\max \left\{0, \tau-\tau_{r-d t}^{*}\right\}\right) \leq \tilde{\phi}_{W}(\tau) .
$$

In other words, we need to make sure that the decrease in value of the function $\phi_{W}$ over any time interval of length smaller or equal to $\tau_{r-d t}^{*}$ time units is never larger than a factor $e^{-\left(\varepsilon^{2}-\delta\right) \tau_{\text {miati }}} \lambda^{2}$, i.e., it should hold that

$$
\frac{\tilde{\phi}_{W}\left(\tau_{1}\right)}{\tilde{\phi}_{W}\left(\tau_{2}\right)} \leq e^{\left(\varepsilon^{2}-\delta\right) \tau_{\text {miati }}} \frac{1}{\lambda^{2}}
$$

for any $\tau_{1}, \tau_{2} \in\left[0, \tau_{\text {mati }}\right]$ with $0 \leq \tau_{2}-\tau_{1} \leq \tau_{r-d t}^{*}$. Unfortunately, this condition cannot be directly verified for the function $\phi_{W}$ as it involves a numerical check for an infinite amount of points. Therefore, to verify (29) we aim to bound the derivative of the function $\tilde{\phi}_{W}$ from below by considering the following lemma, whose proof is given in Appendix A.

Lemma 1. When the function $\tilde{\phi}_{W}:\left[0, \tau_{\text {mati }}\right] \rightarrow \mathbb{R}_{\geq 0}$ is such that for all $\tau \in\left[0, \tau_{\text {mati }}\right]$ it holds that

$$
\frac{\mathrm{d}}{\mathrm{d} \tau} \tilde{\phi}_{W}(\tau) \geq \lambda_{W} \tilde{\phi}_{W}(\tau)
$$

with

$$
\lambda_{W}:=\frac{\ln \left(\lambda^{2}\right)-\left(\varepsilon^{2}-\delta\right) \tau_{\text {miati }}}{\tau_{r-d t}^{*}},
$$

then $\tilde{\phi}_{W}$ satisfies (29) (and therefore also (28)).

Hence, from Lemma 1 it follows that, when (30) holds with (31), we obtain, in view of (26) and (27), that

$$
\begin{aligned}
U(G(\xi)) & \leq \phi_{V}(\tau) V(x)+\gamma \frac{1}{\lambda^{2}} \phi_{W}(\tau) \lambda^{2} W^{2}(\kappa, e) \\
& \leq U(\xi),
\end{aligned}
$$

and, hence, $(11 \mathrm{c})$ is also satisfied.

Based on (22) and (32), we thus conclude by application of Theorem 1 that, for the choice of Lyapunov function $U$ given by (16) with (20) and (21) (cf. (25) with (20) and (24)), the set $\mathcal{E}$ is UGES for the NCS, under the condition that (17b) and (30) hold. We therefore now need to identify conditions on the RADT $\tau_{r-d t}^{*}$ and the MATI $\tau_{m a t i}$ such that (17b) and (30) are satisfied.

Remark 5. There exists quite some similarity in the Lyapunov function constructions of (25) and (19) as both of them satisfy the Lyapunov conditions (11) and consist of a nondecreasing exponential function multiplied with a nonlinear function. However, while the function $\mathcal{U}$ as in (19) satisfies the 'nonmonotonic' Lyapunov conditions (18), the function $\tilde{U}(\xi)$ := $V(x)+\gamma \tilde{\phi}_{W}(\tau) W^{2}(\kappa, e)$ as in (25) satisfies the Lyapunov conditions (11) (when (17b) and (30) hold). Note here also that the function $\tilde{U}$ is essentially the same as the Lyapunov function as used in [12, Proof of Theorem 1]. This implies that the inclusion of the RADT condition does not inherently change the behavior of the Lyapunov function with respect to [12], making it indeed more natural to consider the Lyapunov construction from (25) in the analysis for NCSs.

Remark 6. We would like to have that the value for $\left|\lambda_{W}\right|$ in (30) is as large as possible, which again underlines the tradeoff between the values for $\tau_{r-d t}^{*}$ and $\tau_{m a t i}$ as discussed in Remark 1. In particular, from (30) it follows that when the value for $\tau_{r-d t}^{*}$ is taken larger, $\left|\lambda_{W}\right|$ becomes smaller (i.e., closer to zero), and, hence, (30) imposes a stricter condition on the function $\tilde{\phi}_{W}$, making it more difficult to be satisfied for 'large' values of $\tau_{\text {mati }}$. Fortunately, as $\frac{\mathrm{d}}{\mathrm{d} \tau} \tilde{\phi}_{W}(\tau)$ will always have a finite value for all $\tau \in\left[0, \tau_{\text {mati }}\right]$ as a result of (17), we have that for a small enough value for $\tau_{r-d t}^{*}>\tau_{\text {miati }}$ (and, therefore, also for $\tau_{\text {miati }}>0$ ), it can be realized that $\lambda_{W}<0$ is small enough and, therefore, that there always exists some finite $\tau_{\text {mati }} \geq \tau_{r-d t}^{*} \geq \tau_{\text {miati }}$ for which (30) holds.

Remark 7. Having $\tau_{\text {miati }}=0$ (i.e., essentially zero) in the value for $\lambda_{W}$ (and, hence, in (28) and (29)) is already sufficient for (32) (cf. (11c)) to be satisfied. As such, similar to [22], it directly follows that exploiting knowledge on the existence of a positive MIATI leads to higher values for the MATI (when $\left.\delta<\varepsilon^{2}\right)$ as in this case $\left|\lambda_{W}\right|$ is larger, see also Remark 6.

\section{Ensuring (17b) and (30)}

Combining (24) and (30), it follows that (30) can be rewritten for all $\tau \in\left[0, \tau_{\text {mati }}\right]$ as

$$
-\gamma\left(\tilde{\phi}_{W}^{2}(\tau)+1\right)-\left(2 L+\varepsilon^{2}\right) \tilde{\phi}_{W}(\tau) \geq \lambda_{W} \tilde{\phi}_{W}(\tau)
$$

or, equivalently, as

$$
\gamma \tilde{\phi}_{W}^{2}(\tau)+\left(2 L+\varepsilon^{2}+\lambda_{W}\right) \tilde{\phi}_{W}(\tau)+\gamma \leq 0,
$$

which is a polynomial inequality of degree 2 in $\tilde{\phi}_{W}$ for which we can consider the following well-known lemma.

Lemma 2. For $a, b, c \in \mathbb{R}$ with $a>0$, we have that $a x^{2}+b x+c \leq$ 0 if and only if $c<\frac{b^{2}}{4 a}$, and

$$
-\frac{1}{2}\left(\sqrt{\frac{b^{2}-4 a c}{a^{2}}}+\frac{b}{a}\right) \leq x \leq \frac{1}{2}\left(\sqrt{\frac{b^{2}-4 a c}{a^{2}}}-\frac{b}{a}\right) .
$$

Hence, finding the (maximal) value for $\tau_{\text {mati }}$ such that (33) (cf. (30)) holds is now equivalent to verifying for which (maximal) value for $\tau_{m a t i}$ the conditions of Lemma 2 are satisfied for a given $\tau_{\text {miati }}>0$ and $\tau_{r-d t}^{*}>\tau_{\text {miati }}$, where we define

$$
a=\gamma, \quad b=2 L+\varepsilon^{2}+\lambda_{W}, \quad \text { and } c=\gamma .
$$


As such, using Lemma 2, we have that the following condition (cf. $c<\frac{b^{2}}{4 a}$ ) should hold

$$
\begin{aligned}
\gamma<\frac{\left(2 L+\varepsilon^{2}+\lambda_{W}\right)^{2}}{4 \gamma} & \Leftrightarrow 4 \gamma^{2}<\left(2 L+\varepsilon^{2}+\lambda_{W}\right)^{2} \\
& \Leftrightarrow\left|2 L+\varepsilon^{2}+\lambda_{W}\right|>2 \gamma .
\end{aligned}
$$

In addition to this result, the bounds in (34) should also be verified for the given values in (35). To this end, we compute using (35)

$$
\sqrt{\frac{b^{2}-4 a c}{a^{2}}}=\frac{1}{\gamma} \sqrt{\left(2 L+\varepsilon^{2}+\lambda_{W}\right)^{2}-4 \gamma^{2}}
$$

(which is always positive as a result of (36)) and

$$
\frac{b}{a}=\frac{1}{\gamma}\left(2 L+\varepsilon^{2}+\lambda_{W}\right) .
$$

Hence, we can now explicitly compute the lower and upper bounds for the function $\tilde{\phi}_{W}$ based on (34) such that (33) (cf. (30)) holds. In particular, based on (34) and (37), we have that for all $\tau \in\left[0, \tau_{\text {mati }}\right]$ it should hold that

$$
\underline{\phi}_{W} \leq \tilde{\phi}_{W}(\tau) \leq \overline{\tilde{\phi}}_{W}
$$

with

$$
\begin{aligned}
& \tilde{\tilde{\phi}}_{W}:=-\frac{1}{2 \gamma}\left(\sqrt{\left(2 L+\varepsilon^{2}+\lambda_{W}\right)^{2}-4 \gamma^{2}}+\left(2 L+\varepsilon^{2}+\lambda_{W}\right)\right) \\
& \overline{\tilde{\phi}}_{W}:=\frac{1}{2 \gamma}\left(\sqrt{\left(2 L+\varepsilon^{2}+\lambda_{W}\right)^{2}-4 \gamma^{2}}-\left(2 L+\varepsilon^{2}+\lambda_{W}\right)\right) .
\end{aligned}
$$

However, in order to have a strictly positive upper bound $\overline{\tilde{\phi}}_{W}$ following from (17b), it must, in addition to (36), hold that

$$
2 L+\varepsilon^{2}+\lambda_{W}<0 \text {. }
$$

Fortunately, we can now obtain that both conditions (36) and (39) are satisfied when

$$
\begin{aligned}
& 2 L+\varepsilon^{2}+\frac{\ln \left(\lambda^{2}\right)-\left(\varepsilon^{2}-\delta\right) \tau_{\text {miati }}}{\tau_{r-d t}^{*}}<-2 \gamma \\
\Rightarrow & \tau_{r-d t}^{*}<\left|\frac{\ln \left(\lambda^{2}\right)-\left(\varepsilon^{2}-\delta\right) \tau_{\text {miati }}}{2 \gamma+2 L+\varepsilon^{2}}\right| .
\end{aligned}
$$

Moreover, as a direct result of (40) (cf. (39)), it now also follows that the lower bound on $\tilde{\phi}_{W}$ is strictly positive, i.e., $\tilde{\phi}_{W}>0$. This implies that when the RADT $\tau_{r-d t}^{*}$ satisfies (40), then also condition (17b) holds. In addition, when we choose $\tilde{\phi}_{W}(0)=\tilde{\phi}_{W}$, we can directly compute the value for $\tau_{m a t i}$ as being the point in time at which

$$
\tilde{\phi}_{W}\left(\tau_{m a t i}\right)=\tilde{\phi}_{W}
$$

since $\tilde{\phi}_{W}$ is a strictly decreasing function, where, in addition, the MATI $\tau_{m a t i}$ has the highest possible value it can attain while satisfying (33). Moreover, as

$$
\begin{aligned}
\left(\overline{\tilde{\phi}}_{W}\right)^{-1} & =2 \gamma \frac{1}{\sqrt{\left(2 L+\varepsilon^{2}+\lambda_{W}\right)^{2}-4 \gamma^{2}}-\left(2 L+\varepsilon^{2}+\lambda_{W}\right)} \\
& =2 \gamma \frac{\sqrt{\left(2 L+\varepsilon^{2}+\lambda_{W}\right)^{2}-4 \gamma^{2}}+\left(2 L+\varepsilon^{2}+\lambda_{W}\right)}{\left(2 L+\varepsilon^{2}+\lambda_{W}\right)^{2}-4 \gamma^{2}-\left(2 L+\varepsilon^{2}+\lambda_{W}\right)^{2}} \\
& =-\frac{1}{2 \gamma}\left(\sqrt{\left(2 L+\varepsilon^{2}+\lambda_{W}\right)^{2}-4 \gamma^{2}}+\left(2 L+\varepsilon^{2}+\lambda_{W}\right)\right) \\
& =\underline{\tilde{\phi}}_{W},
\end{aligned}
$$

we can additionally use the result from [12, Claim 1] (see also Appendix B) to obtain an explicit expression for the MATI.

In summary, we have thus identified conditions (i.e., (36), (38) and (39)) under which the inequality (30) indeed holds and (17b) is satisfied, and, in particular, we can now compute the pairs $\left(\tau_{r-d t}^{*}, \tau_{m a t i}\right)$ by means of (40) and (41) for which the NCS is UGES. As such, we can state our main result.

\section{Main Result}

When we collect the results as found above, we can state the following theorem.

Theorem 2. Under Assumption 1 and for a given value of the MIATI $\tau_{\text {miati }}>0$, if the RADT $\tau_{r-d t}^{*} \geq \tau_{\text {miati }}$ satisfies for some constants $0<\delta<\varepsilon^{2}$ and $\psi:=2 L+\varepsilon^{2}$ the bound

$$
\tau_{r-d t}^{*}<\left|\frac{\ln \left(\lambda^{2}\right)-\left(\varepsilon^{2}-\delta\right) \tau_{\text {miati }}}{2 \gamma+\psi}\right|,
$$

and if the MATI $\tau_{m a t i} \geq \tau_{r-d t}^{*}$ satisfies the bound $\tau_{m a t i} \leq$ $T\left(\lambda, \tau_{r-d t}^{*}\right)$ where

$$
T\left(\lambda, \tau_{r-d t}^{*}\right)= \begin{cases}\frac{2}{\psi r} \arctan \left(\frac{r(1-\sigma)}{2 \frac{\sigma}{1+\sigma}\left(\frac{2 \gamma}{\psi}-1\right)+1+\sigma}\right), & 2 \gamma>\psi \\ \frac{2}{\psi} \frac{1-\sigma}{1+\sigma}, & 2 \gamma=\psi \\ \frac{2}{\psi r} \operatorname{arctanh}\left(\frac{r(1-\sigma)}{2 \frac{\sigma}{1+\sigma}\left(\frac{2 \gamma}{\psi}-1\right)+1+\sigma}\right), & 2 \gamma<\psi\end{cases}
$$

with $\sigma:=-\frac{1}{2 \gamma}\left(\sqrt{\left(\psi+\lambda_{W}\right)^{2}-4 \gamma^{2}}+\left(\psi+\lambda_{W}\right)\right)>0, \lambda_{W}:=$ $\frac{\ln \left(\lambda^{2}\right)-\left(\varepsilon^{2}-\delta\right) \tau_{\text {miati }}}{\tau_{r-d t}^{*}}$, and $r:=\sqrt{\left|\left(\frac{2 \gamma}{\psi}\right)^{2}-1\right|}$, then the set $\mathcal{E}$ given by (10) is UGES for the hybrid system (9).

Based on the explicit bounds presented in Theorem 2, the value for the MATI $\tau_{\text {mati }}$ indeed depends on the value for the RADT $\tau_{r-d t}^{*}$ through $\lambda_{W}$, as expected based on Remarks 1 and 6. In particular, the value for $\tau_{m a t i}$ increases when the value for $\left|\lambda_{W}\right|$ increases (as the value for $\sigma$ decreases in this case, i.e., becomes closer to 0), which corresponds to having a smaller value for $\tau_{r-d t}^{*}$. As such, choosing $\tau_{r-d t}^{*}>\tau_{\text {miati }}$ as small as possible (i.e., essentially equal to the value for the MIATI) will always give the highest possible value for the MATI, while for increasing values of $\tau_{r-d t}^{*}$ we have that $\tau_{\text {mati }}$ becomes smaller. This trade-off between the values for the RADT and the MATI will also be evident in the Section $\mathrm{V}$, where Theorem 2 will be applied to a numerical example to illustrate the application of our results by obtaining for each feasible $\mathrm{RADT}^{3} \tau_{r-d t}^{*}$ its corresponding maximal value for the MATI $\tau_{m a t i} \geq \tau_{r-d t}^{*}$.

The above observations and reasoning, however, also have some consequences for the maximal attainable improvement of the MATI and for the so-called sampled-data (SD) case, as we will show first in the next subsection.

Remark 8. To obtain the highest possible values for the pair $\left(\tau_{r-d t}^{*}, \tau_{m a t i}\right)$, we will take, in practice, $\delta>0$ as small as possible (i.e., essentially zero) since this results in the highest

\footnotetext{
${ }^{3}$ By a feasible $\tau_{r-d t}^{*}$, we mean a RADT for which there exists a MATI $\tau_{\text {mati }}>\tau_{r-d t}^{*}$ such that UGES for the NCS is guaranteed.
} 


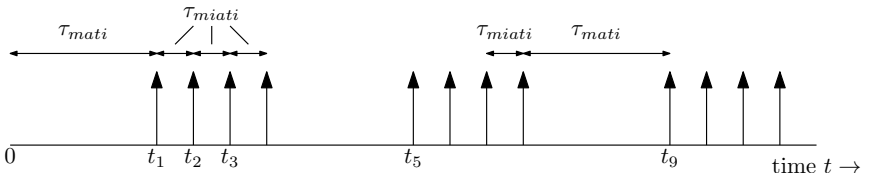

Fig. 2. Illustration of a periodic sequence of transmission times $t_{j} \in \mathbb{R}_{\geq 0}$, $j \in \mathbb{N}$, consisting of one transmission after $\tau_{\text {mati }}$ time units, immediately succeeded by a number of consecutive transmissions each after $\tau_{\text {miati }}$ time units.

possible value for $\left|\lambda_{W}\right|$ as desired, see Remark 6. Moreover, it follows from both the bounds (42) and (43) for $\tau_{r-d t}^{*}$ and $\tau_{\text {mati }}$, respectively, that they are maximal when $\gamma$ is taken as small as possible (while satisfying (15)). Similarly, it also follows that having $\varepsilon$ large would in general lead to higher values of the RADT and the MATI, especially when the value for the MIATI is large, see also Remark 7. However, this would also lead to the value of $\gamma$ being larger due to constraint (15), which results in lower values for the RADT and the MATI. Therefore, we will in general take $\varepsilon$ small, however, it is this balancing effect that might give in some specific cases better results for larger values of $\varepsilon$.

Remark 9. Throughout the section, several design choices are made, e.g., constructing a Lyapunov function satisfying (11) instead of (18) or verifying (29) by means of Lemma 1. Although these choices were educated and helped in trimming the number of free parameters, they still might introduce some conservatism in the results. Nevertheless, the numerical example in Section $\mathrm{V}$ shows that we can obtain a significant improvement for the MATI with respect to the results in [12] and [22]. Moreover, we made these choices to obtain a much cleaner and natural analysis, and more transparent conditions.

\section{E. Upper Bounding the MATI and the Sampled-Data Case}

Based on the results from Theorem 2 and [12], it is possible to obtain an upper bound on the value of $T\left(\lambda, \tau_{r-d t}^{*}\right)$ for some $\lambda>0$, depending on the RADT $\tau_{r-d t}^{*}$. This provides valuable insights with respect to the maximal improvement of the MATI we can achieve using Theorem 2, which are especially interesting for the so-called sampled-data (SD) case (see Remark 14). To substantiate this, we will first make use of the following reasoning.

Consider for a given $\tau_{\text {miati }}, \tau_{r-d t}^{*}$, and $\tau_{m a t i}=T\left(\lambda, \tau_{r-d t}^{*}\right)$, satisfying Theorem 2 , the possible periodic sequence of transmission times as depicted in Fig. 2, consisting of one transmission after $\tau_{m a t i}$ time units, immediately succeeded by $N-1$ consecutive transmissions each after $\tau_{\text {miati }}$ time units with

$$
N:=\left\lceil\frac{\tau_{\text {mati }}-\tau_{\text {miati }}}{\tau_{r-d t}^{*}-\tau_{\text {miati }}}\right\rceil,
$$

and repeated with period $\tau_{\text {mati }}+(N-1) \tau_{\text {miati }}$ afterwards. This sequence of transmission times satisfies (7) with an average transmission interval of

$$
\frac{1 \cdot \tau_{\text {mati }}+(N-1) \cdot \tau_{\text {miati }}}{N} \leq \tau_{r-d t}^{*} .
$$

When we now take $\tau_{\text {miati }}=0$, the sequence of transmission times from Fig. 2 reduces to having

$$
N=\left\lceil\frac{\tau_{\text {mati }}}{\tau_{r-d t}^{*}}\right\rceil
$$

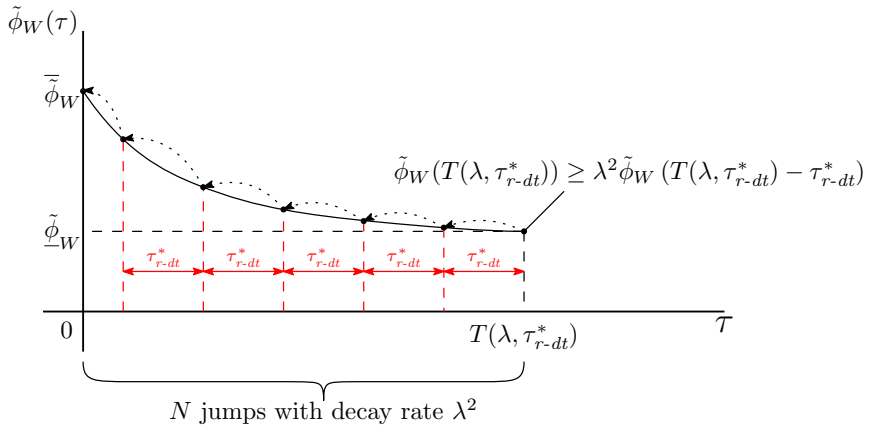

(a) Illustration of the evoluation of the function $\tilde{\phi}_{W}$ from (24) in the situation that each time $N$ transmissions (cf. jumps) occur after MATI time units with $\tau^{+}=\max \left\{0, \tau-\tau_{r-d t}^{*}\right\}$. The MATI is determined by the point in time at which (41) holds.

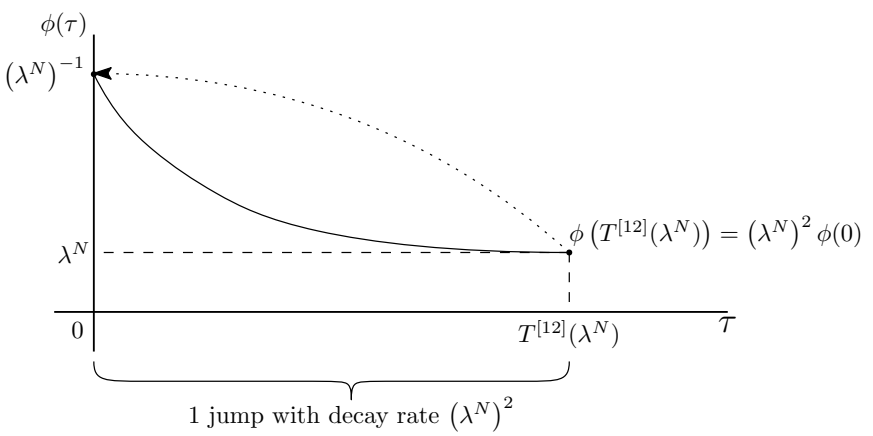

(b) Illustration of the evolution of the function $\phi$ from [12, Claim 1] in the situation that one transmission (cf. jump) occurs after each MATI time units with $\tau^{+}=0$. Hence, the MATI $T^{[12]}\left(\lambda^{N}\right)$ is determined by the point in time at which (46) holds with $\phi(0)=\left(\lambda^{N}\right)^{-1}$.

Fig. 3. Illustrations of the evolutions of the functions $\phi$ as in [12, Claim 1] and $\tilde{\phi}_{W}$ from (24) for the transmission sequence of Fig. 2 with $\tau_{\text {miati }}=0$.

transmissions after $\tau_{m a t i}$ time units, i.e., the error update (8) based on the scheduling protocol is applied $N$ times at one time instant. The consequences of this scenario for the function $\tilde{\phi}_{W}$ are illustrated in Fig. 3(a).

Remark 10. In the (hypothetical) case that $\tau_{\text {miati }}=0$, Theorem 2 can still be applied to conclude stability when nonZenoness is assumed, i.e., when for the transmission times $t_{j}, j \in \mathbb{N}$, it holds that $t_{j} \rightarrow \infty$ when $j \rightarrow \infty$. As for the transmission sequence of Fig. 2 there always exists a finite number of transmissions after $\tau_{\text {mati }}$ time units, this property is indeed guaranteed.

As depicted in Fig. 3(a) and discussed in Section IV-C, the value for $\tau_{\text {mati }}=T\left(\lambda, \tau_{r-d t}^{*}\right)$ is determined by the point in time at which (41) holds. Moreover, in the situation of the periodic transmission sequence of Fig. 2 with $\tau_{\text {miati }}=0$, we have at this value of the MATI $N$ consecutive transmissions, where for each transmission (cf. jump of the hybrid system) (29) holds with $\tau_{\text {miati }}=0$. As a result of this latter observation, it follows that

$$
\tilde{\phi}_{W}\left(T\left(\lambda, \tau_{r-d t}^{*}\right)\right) \geq\left(\lambda^{2}\right)^{N} \tilde{\phi}_{W}(0),
$$

see also Fig. 3(a), where $\tilde{\phi}_{W}$ is the solution to the differential equation (24) for the given $\lambda$ and $\tau_{r-d t}^{*}$ with $\tau_{\text {miati }}=0$. Observe also that

$$
\tilde{\phi}_{W}(0)=\overline{\tilde{\phi}}_{W}=\left(\underline{\tilde{\phi}}_{W}\right)^{-1}=\left(\tilde{\phi}_{W}\left(T\left(\lambda, \tau_{r-d t}^{*}\right)\right)\right)^{-1},
$$


implying in combination with (41) and (44) that, for the value of $T\left(\lambda, \tau_{r-d t}^{*}\right)$, we always have that

$$
\tilde{\phi}_{W}\left(T\left(\lambda, \tau_{r-d t}^{*}\right)\right) \geq \lambda^{N} .
$$

Using now that $\tilde{\phi}_{W}$ is the solution to (24), it follows that we can upper bound the value $T\left(\lambda, \tau_{r-d t}^{*}\right)$ by using the results from [12]. In particular, as illustrated in Fig. 3(b) and discussed in [12], the value for the MATI for an UGES scheduling protocol with decay rate $\lambda^{N}$ (see also (47) below), which we denote by $T^{[12]}\left(\lambda^{N}\right)$, is determined by the point in time for which it holds that

$$
\phi\left(T^{[12]}\left(\lambda^{N}\right)\right)=\left(\lambda^{N}\right)^{2} \phi(0),
$$

where the function $\phi$ satisfies the differential equation (24) for $\varepsilon=0$ with $^{4} \phi(0)=\left(\lambda^{N}\right)^{-1}$, see the proof of [12, Theorem 1]. Indeed, for the transmission sequence as described above, we have that (12b) is applied $N$ times, resulting in

$$
W\left(h^{N}(e, \kappa), \kappa+N\right) \leq \lambda^{N} W(e, \kappa),
$$

where $h^{N}$ is determined by the recurring sequence

$$
h^{l}(e, \kappa)=h\left(h^{l-1}(e, \kappa), \kappa+l-1\right), \quad l \in\{2,3, \ldots, N\}
$$

where for $l=1$ we have that $h^{1}(e, \kappa)=h(e, \kappa)$. In other words, the above described scenario (with $\tau_{\text {miati }}=0$ ) is equivalent to the situation in which we have one transmission after $\tau_{\text {mati }}$ time units with an UGES scheduling protocol for which (12b) is satisfied with $\lambda^{N}$, which corresponds to the setting of [12, Theorem 1]. Using, in addition, the result from [12, Claim 1], it follows that

$$
\phi\left(T^{[12]}\left(\lambda^{N}\right)\right)=\lambda^{N} .
$$

Since now for any $\varepsilon>0$ we have that the function $\tilde{\phi}_{W}$ decreases faster than the function $\phi$, it directly follows from comparing (45) and (48) that

$$
T\left(\lambda, \tau_{r-d t}^{*}\right) \leq T^{[12]}\left(\lambda^{N}\right) .
$$

Based on this reasoning, we have the following result.

Proposition 2. Consider the hybrid system (9) with the MIATI $\tau_{\text {miat } i}=0$ and suppose Assumption 1 holds with $0<\lambda<1$. For a given $R A D T \tau_{r-d t}^{*}$ and MATI $\tau_{\text {mati }} \leq T\left(\lambda, \tau_{r-d t}\right)$ computed by (43) for which the set $\mathcal{E}$ is UGES according to Theorem 2, we have that

$$
T\left(\lambda, \tau_{r-d t}^{*}\right) \leq T^{[12]}\left(\lambda^{N}\right) \text { with } N=\left\lceil\frac{T\left(\lambda, \tau_{r-d t}^{*}\right)}{\tau_{r-d t}^{*}}\right\rceil
$$

where $T^{[12]}\left(\lambda^{N}\right)$ denotes the MATI as computed by [12, Theorem 1] with an UGES scheduling protocol for which (12b) is satisfied for decay rate $\lambda^{N}$ (cf. (47)).

Proposition 2 provides an upper bound on the value that the MATI can attain as a function of the RADT (in the case that $\tau_{\text {miati }}=0$ ). This allows us to make several important

\footnotetext{
${ }^{4}$ Although it was not shown or mentioned in [12], choosing the initial condition as $\phi(0)=\left(\lambda^{N}\right)^{-1}$ is actual the 'optimal' choice since it results in the largest value for $T^{[12]}\left(\lambda^{N}\right)$ for which (46) is satisfied. To substantiate this, we provide in Appendix B a note on the 'optimality' of the result from [12].
}

observations. Firstly, when $\tau_{r-d t}^{*}=T\left(\lambda, \tau_{r-d t}^{*}\right)\left(=\tau_{m a t i}\right)$ in Theorem 2 (i.e., $N=1$ in Proposition 2), we have that the upper bound $T^{[12]}\left(\lambda^{N}\right)$ on the MATI in (49) is actually equal to the value of the MATI as computed in [12] for the NCS setup of (2) and (3) with (4). Secondly, it follows that, since $\lambda<1$ and in view of Remark 11, the upper bound on the MATI converges to the value $\lim _{\lambda \downarrow 0} T^{[12]}(\lambda)$ when $N \rightarrow \infty$ as $\lambda^{N} \rightarrow 0$ in this case, see also Remark 12. Hence, the maximal value for the MATI we can obtain using Theorem 2 is bounded by the value for the MATI as computed using [12, Theorem 1] in the SD case with $\lambda \rightarrow 0$ in view of Remark 14, see also the result of [13] where the value for the MATI is computed along the lines of [12] with $\lambda=0$. This also implies our final conclusion, given by the following result.

Corollary 1. Consider the hybrid system (9) and suppose Assumption 1 holds with $0<\lambda<1$. For any given MIATI $\tau_{\text {miati }}>0, R A D T \tau_{r-d t}^{*}$, and MATI $\tau_{\text {mat } i} \leq T\left(\lambda, \tau_{r-d t}\right)$ for which the set $\mathcal{E}$ is UGES according to Theorem 2, we have that

$$
T\left(\lambda, \tau_{r-d t}^{*}\right) \leq \lim _{\tau_{r-d t}^{*} \downarrow 0} T\left(\lambda, \tau_{r-d t}^{*}\right)=\lim _{\lambda \downarrow 0} T\left(\lambda, \tau_{r-d t}^{*}\right) \leq T^{[13]}(0)
$$

where $T^{[13]}(0)$ denotes the MATI as computed by [13, Theorem 2] for the $S D$ protocol with $\lambda=0$.

Corollary 1 implies that in the SD case actually no improvement of the MATI can be obtained using Theorem 2 with respect to the results of [13]. Moreover, in this case the value for the MATI will be the same for any value for the RADT. This can also directly be linked to condition (28), which is in the SD case always satisfied as $\lambda=0$. In particular, computing the MATI reduces in this case to determining the point in time at which the Lyapunov function $U$ is no longer guaranteed to be strictly positive, which coincides with the point in time at which the function $\tilde{\phi}_{W}$ is no longer strictly positive (cf. (17b) fails to hold). As such, in order to obtain an improvement of the MATI when exploiting a RADT condition in the SD case, we must either exclude the situation in which the MIATI is allowed to be essentially zero (as mentioned above) or have some indication regarding the spread of the transmissions over time. However, this is left for future work.

Remark 11. Using the results from [12], it follows that

$$
T^{[12]}\left(\lambda_{1}\right)>T^{[12]}\left(\lambda_{2}\right)
$$

when $\lambda_{1}<\lambda_{2}<1$. As such, since $\lambda<1$ we have that (49) actually holds for any

$$
N \geq\left\lceil\frac{T\left(\lambda, \tau_{r-d t}^{*}\right)}{\tau_{r-d t}^{*}}\right\rceil .
$$

Note also that, similarly, from (43) it follows that

$$
T\left(\lambda_{1}, \tau_{r-d t, 1}^{*}\right)>T\left(\lambda_{2}, \tau_{r-d t, 2}^{*}\right)
$$

when $\lambda_{1}<\lambda_{2}<1$ and/or $\tau_{r-d t, 1}^{*}<\tau_{r-d t, 2}^{*}$. Indeed, a lower value for $\lambda / \tau_{r-d t}^{*}$ results in a higher value for $\lambda_{W}$, and, hence, for a lower value of $\sigma$, which gives a higher value $T\left(\lambda, \tau_{r-d t}^{*}\right)$.

Remark 12. Following from Remark 11, observe that the limit

$$
\lim _{\lambda \downarrow 0} T^{[12]}(\lambda)=T^{[12]}(0) \quad\left(:=T^{[13]}(0)\right)
$$


exists due to $T^{[12]}(\lambda)$ being continuous in $\lambda=0$ as a result of the monotonicity property (50) (see also the proof of [13, Theorem 1]). Similarly, the limits in Corollary 1 also exist as $T\left(\lambda, \tau_{r-d t}^{*}\right)$ is continuous in both $\lambda$ and $\tau_{r-d t}^{*}$ as a result of the monotonicity property (51).

Remark 13. Note that the upper bound $T^{[12]}\left(\lambda^{N}\right)$ in Proposition 2 can only be computed a posteriori, i.e., we first need to compute the value $T\left(\lambda, \tau_{r-d t}^{*}\right)$ in order to determine the value for $N$ in (49). However, the existence of such an upper bound provides us with valuable information concerning the limit cases $N=1$ and $N \rightarrow \infty$. Indeed, the bounds $T^{[12]}(\lambda)$ in Proposition 2 and $T^{[13]}(0)$ in Corollary 1 can be computed a priori, indicating which improvements we can attain.

Remark 14. Sampled-data (SD) NCSs are systems for which all the nodes in the network are updated simultaneously at a transmission time. That is, the scheduling protocol function $h: \mathbb{N}_{0} \times \mathbb{R}^{m_{e}} \rightarrow \mathbb{R}^{m_{e}}$ in (3) is in this case simply given by $h(\kappa, e)=0$, implying that an update of the networked values results in the network-induced error being set to zero, i.e., $e^{+}=0$ in (8), see also [11] or [13]. As a result of this property, we can take $W(\kappa, e)=|e|$ in Assumption 1, implying that (12b) is satisfied for any $\lambda$, including $\lambda=0$ (since $W(\kappa+$ $1, h(\kappa, e))=|h(\kappa, e)|=0)$.

\section{NuMERICAL EXAMPLE}

In [27], we have already shown the effectiveness of our results on a linear example leading to an improvement of the MATI of up to $484 \%$ in comparison to the results of [12], [22]. To further substantiate the usefulness of exploiting an RADT condition for NCSs, we consider in this section the nonlinear example of a single-link robot arm as described in [18], whose dynamics are modeled by

$$
\mathcal{P}:\left\{\begin{aligned}
\dot{x}_{p 1} & =x_{p 2} \\
\dot{x}_{p 2} & =-a \sin \left(x_{p 1}\right)+b \hat{u} \\
y & =\left(x_{1}, x_{2}\right)
\end{aligned}\right.
$$

and which is controlled by the static state feedback controller

$$
\mathcal{C}: u=b^{-1}\left(\sin \left(\hat{x}_{p 1}\right)-\hat{x}_{p 1}-\hat{x}_{p 2}\right)
$$

with $\hat{x}_{p 1}, \hat{x}_{p 2}$, and $\hat{u}$ the networked values of $x_{p 1}, x_{p 2}$, and $u$, respectively. Hence, from this system configuration it follows that the network-induced error is given by $e=\left(e_{y}, e_{u}\right)=$ $\left(e_{x_{p 1}} \cdot e_{x_{p 2}}, e_{u}\right)=\left(\hat{x}_{p 1}-x_{p 1}, \hat{x}_{p 2}-x_{p 2}, \hat{u}-u\right)$ The number of nodes in the network is $\ell=3$. As a result, we can model the closed-loop dynamics as a hybrid system of the form (9) where $f(x, e)=\left(x_{p 2},-a \sin \left(x_{p 1}\right)+b e_{u}+a \sin \left(x_{p 1}+e_{x_{p 1}}\right)-\right.$ $\left.x_{p 1}-e_{x_{p 1}}-x_{p 2}-e_{x_{p 2}}\right)$ and $g(x, e)=-(f(x, e), 0)$.

For this nonlinear example we consider the TOD protocol with $W(\kappa, e)=|e|$, which satisfies (12) and has the property that $\left|\frac{\partial W(\kappa, e)}{\partial e}\right| \leq M$ for all $\kappa \in \mathbb{N}_{0}$ with $\lambda=\sqrt{\ell-1 / \ell}=\frac{1}{3} \sqrt{6}$ and $\underline{\alpha}_{W}=\bar{\alpha}_{W}=M=1$, see, e.g., [21], [22]. Combining this with the fact that $|g(x, e)|=|f(x, e)| \leq\left|x_{p 2}\right|+(1+a)\left|e_{x_{p 1}}\right|+$ $b\left|e_{u}\right|+\left|x_{p 1}+x_{p 2}\right|+\left|e_{x_{p 2}}\right| \leq\left|x_{p 2}\right|+\left|x_{p 1}+x_{p 2}\right|+D|e|$, where $D=\sqrt{3} \max \{1+a, b\}$, we obtain that (13) is satisfied for $L=M D$ and $H(x)=M\left(\left|x_{p 2}\right|+\left|x_{p 1}+x_{p 2}\right|\right)$.
To satisfy Assumption 1, we choose $V(x)=\alpha x_{p 1}^{2}+$ $\beta x_{p 1} x_{p 2}+\theta x_{p 2}^{2}$ where $\alpha, \beta, \theta>0$ will be chosen such that (14) holds. Writing $a \sin \left(x_{p 1}\right)-a \sin \left(x_{p 1}+e_{x_{p 1}}\right)=\bar{a} e_{x_{p 1}}$ with varying parameter $\bar{a} \in[-a, a]$ and applying the fact that $x y \leq \frac{\eta}{2} x^{2}+\frac{1}{2 \eta} y^{2}$ for $x, y \in \mathbb{R}_{\geq 0}$ and $\eta>0$, we obtain that $\langle\nabla V(x), f(x, e)\rangle \leq-\beta x_{p 1}^{2}-(2 \theta-\beta) x_{p 2}^{2}+(2 \alpha-2 \theta-$ B) $x_{p 1} x_{p 2}+\frac{1}{2 \eta}\left(2 \theta x_{p 2}+\beta x_{p 1}\right)^{2}+\frac{1}{2} \eta D^{2}|e|^{2}$ for some constant $\eta>0$, see also [18]. Therefore, if we ensure that (14) holds and $-\tilde{\varepsilon}|x|^{2}-H^{2}(x) \leq-\beta x_{p 1}^{2}-(2 \theta-\beta) x_{p 2}^{2}+(2 \alpha-2 \theta-\beta) x_{p 1} x_{p 2}+$ $\frac{1}{2 \eta}\left(2 \theta x_{p 2}+\beta x_{p 1}\right)^{2}$ with $\tilde{\varepsilon}>0$, then Assumption 1 is satisfied for $\gamma=\sqrt{\frac{1}{2} \eta D^{2}}$ and $\varepsilon=\tilde{\varepsilon} \underline{\alpha}_{V}$. Observe that this latter condition can be written as $x^{T}(P+\tilde{\varepsilon} I) x \leq 0$ for all $x \in \mathbb{R}^{2}$ with

$$
\begin{aligned}
& P= \\
& {\left[\begin{array}{cc}
-\beta+M^{2}+\frac{1}{2 \eta} \beta^{2} & \alpha-\theta-1 / 2 \beta+\theta \beta \eta^{-1}+2 M^{2} \\
\alpha-\theta-\frac{1}{2} \beta+\theta \beta \eta^{-1}+2 M^{2} & -2 \theta+\beta+2 \theta^{2} \eta^{-1}+4 M^{2}
\end{array}\right] .}
\end{aligned}
$$

Taking $a=9.81 \cdot 0.5$ and $b=2$ in the plant model, we can now use the Matlab optimization toolbox to minimize the value for $\gamma$ subjected to (14) and $P+\tilde{\varepsilon} I \leq 0$, which yields $\alpha=3.054$, $\beta=1.055, \theta=5.053, \eta=10.115, \tilde{\varepsilon}=10^{-3}$, and $\gamma=23.001$. As such, Theorem 2 can now be used to compute the best values for the pair $\left(\tau_{r-d t}^{*}, \tau_{m a t i}\right)$ such that UGES for the NCS described by (52) is guaranteed. The results for $\tau_{\text {miati }}=10^{-6}$ are given in Fig. 4, along with the obtained value for the MATI using [12, Theorem 1] and the upper bound on the MATI from Proposition 2.

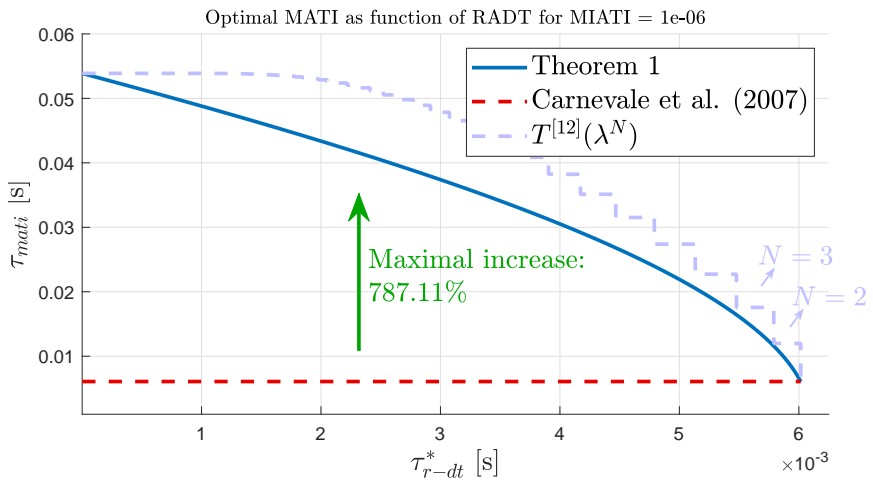

Fig. 4. Computed values for $\left(\tau_{r-d t}^{*}, \tau_{\text {mati }}\right)$ using Theorem 2 for $\tau_{\text {miati }}=$ $10^{-6}$ and the TOD protocol. Also the computed value for the MATI from [12] (see also [18]) is indicated in the figure, along with the upper bound on the MATI $T^{[12]}\left(\lambda^{N}\right)$ from Proposition 2 and the maximal attained improvement with respect to [12].

As shown by Fig. 4, there indeed is a clear trade-off between the RADT and the MATI as discussed by Remarks 1 and 6 . That is, higher values of $\tau_{r-d t}^{*}$ indeed lead to lower values of $\tau_{\text {mati }}$. Moreover, for $\tau_{r-d t}^{*}=\tau_{\text {mati }}$ (i.e., the case that the RADT actually represents a MATI bound, see also Remark 1), we obtain a good approximation of the obtained value for the MATI as in [12] and for $\tau_{r-d t}^{*} \rightarrow 0$ the value for the MATI is indeed bounded by the value for the MATI in the SD case $T^{[12]}(0)$ as computed in [12], see also Proposition 2.

To make now a clearer comparison with the result from [12], some of the results in Fig. 4 have been singled out in Table I. Here, $\tau_{\text {mati }}^{[12]}$ represents the value obtained using the results from [12] (see also [18]) and $\tau_{\text {mati }}$ the value computed using Theorem 2 . It can be directly seen that exploiting a 
TABLE I

THE BOUND ON THE MAXIMAL ALLOWABLE TRANSMISSION INTERVAL FOR $\tau_{\text {miati }}=10^{-6}$ WITH $L=10.228, \lambda=\frac{1}{3} \sqrt{6}, \delta=10^{-6}$, $\varepsilon=10^{-5}$.

\begin{tabular}{|c|c|c|c|c|c|}
\hline \multirow{2}{*}{$\tau_{\text {mati }}^{[12]}$} & \multicolumn{3}{|c|}{$\tau_{\text {mati }}$} & Maximal attained \\
\cline { 2 - 6 } & $\tau_{r-d t}^{*}<0.00567$ & $\tau_{r-d t}^{*}<0.00430$ & $\tau_{r-d t}^{*}<0.00199$ & $\tau_{r-d t}^{*}<2 \cdot 10^{-6}$ & $\begin{array}{c}\text { improvement } \\
\text { of } \tau_{\text {mati }} \text { w.r.t. } \tau_{\text {mati }}^{[12]}\end{array}$ \\
\hline 0.00607 & 0.0137 & 0.0282 & 0.0434 & 0.0539 & $787.11 \%$ \\
\hline
\end{tabular}

RADT condition leads to significantly larger values for the MATI. That is, when we have, for instance, on average a slightly smaller value for the transmission intervals than the MATI from [12] (e.g., $\tau_{r-d t}^{*}<0.00567<\tau_{m a t i}^{[12]}$ ), we can obtain quite a significant improvement of the maximal allowable transmission interval $\tau_{m a t i}$ (e.g., an improvement of $125.6 \%$ in the case of $\tau_{r-d t}^{*}<0.00567$ or even an improvement of $787.11 \%$ in the case of $\tau_{r-d t}^{*}<2 \cdot 10^{-6}$ with respect to the results in [12]). As such, when a RADT is exploited, the time between two transmissions is allowed to be significantly larger than the computed MATI as in [12].

\section{CONCLUSion}

In this paper, we proposed a richer model description of the transmission instants for NCSs by including, next to the presence of a minimal and maximal allowable transmission interval (MIATI/MATI), the specification of a bound on the average allowable transmission interval given by means of a reverse average dwell-time (RADT) condition. Doing so allows for the possibility to model both the worst-case behavior regarding the transmission intervals as analyzed in [8]-[23] and the average case behavior (through the RADT) simultaneously. This leads to significant improvements of the MATI bounds with respect to the results of [12] and [22]. To this end, we made use of the emulation/hybrid systems based approach for NCSs and provided a full Lyapunovbased analysis from which conditions on the RADT and the MATI followed such that UGES for the NCS is guaranteed. Along the way, we also showed that there exists a tradeoff between the RADT and the MATI, which implies that having a smaller RADT directly leads to larger values for the MATI. Additionally, we showed how the maximal attainable improvement of the MATI can be computed a priori, which could be directly linked to the results for the SD case. Finally, for a well-known numerical example with the TOD protocol improvements of the MATI up to $787 \%$ where obtained with respect to the existing results of [12].

The results presented in this paper also have many natural extensions and applications. In particular, for future work, it might be of interest to investigate other possibilities for constructing the functions $\phi_{V}$ and $\phi_{W}$, which would lead to other, perhaps simpler, conditions. Moreover, addressing problems like tracking or state estimation or tailoring the results to the linear case are of high relevance. Finally, extending the work to the event-triggered case could be of interest, where the main difficulty would be to find a hybrid clock that guarantees the RADT condition for event-triggered transmission instants. In any case, we foresee that this novel approach in the analysis of NCSs can possibly inspire even sharper analysis tools.

\section{APPENDIX A \\ PROOFS}

Proof of Proposition 1: The proof consists of two parts, namely showing that each hybrid time domain of a solution $(\tau, s)$ to $(6)$ with initial state set $\left[0, \tau_{m a t i}\right] \times\left(-\infty, \tau_{m a t i}-\right.$ $\left.\tau_{\text {miati }}\right]$ satisfies the conditions (7) and that each hybrid time domain $E$ satisfying (7) is also a hybrid time domain for some solution $(\bar{\tau}, \bar{s})$ to $(6)$ with initial state set $\left[0, \tau_{\text {mati }}\right] \times$ $\left(-\infty, \tau_{\text {mati }}-\tau_{\text {miati }}\right]$.

For the first part, suppose that $(\tau, s)$ is a solution to (6) with initial state set $\left[0, \tau_{\text {mati }}\right] \times\left(-\infty, \tau_{\text {mati }}-\tau_{\text {miati }}\right]$. We observe that for two consecutive jump times $t_{j}$ and $t_{j+1}$, $j \in \mathbb{N}$, we have that $\tau\left(t_{j+1}, j\right) \geq s\left(t_{j+1}, j\right)+\tau_{\text {miati }}$ with $s\left(t_{j+1}, j\right)=s\left(t_{j}, j\right)=\tau\left(t_{j}, j\right)$, implying that it is ensured that two consecutive jumps are at least separated by $\tau_{\text {miati }}$ time units as $\tau\left(t_{j+1}, j\right)-s\left(t_{j+1}, j\right)=t_{j+1}-t_{j}$ due to the dynamics of $\tau$ and $s$. Hence, a solution $(\tau, s)$ indeed satisfies (7a). Moreover, for each $(t, i),(\bar{t}, j) \in \operatorname{dom}(\tau, s)$ with $\bar{t}+j \geq t+i$ we have that, in view of (6),

$$
\tau_{\text {mat } i} \geq \tau(\bar{t}, j)-\tau(t, i) \geq(\bar{t}-t)-(j-i) \tau_{r-d t}^{*}
$$

as $\dot{\tau}=1$ during flows and $\tau$ is at most decreased by $\tau_{r-d t}^{*}$ time units during jumps, see also the proof of [31, Proposition 1.2]. Rearranging the inequality (53) directly gives (7b).

For the second part, suppose that a hybrid time domain $E$ with its sequence of jump times $\left\{t_{j}\right\}_{j \in \mathbb{N}}$ satisfies the conditions given by (7). To construct now a solution $(\bar{\tau}, \bar{s})$ to (6) with the hybrid time domain $E$, we take $\bar{\tau}^{5}(0,0)=0$ and $\bar{s}(0,0) \leq$ $-\tau_{\text {miati }}$ and define $(\bar{\tau}, \bar{s})$ to be the hybrid arc on the hybrid time domain $E$ that satisfies for almost all $t \in\left[t_{j}, t_{j+1}\right], j \in \mathbb{N}_{0}$, with $t_{0}=0$ (see Definition 1a)

$$
\dot{\bar{\tau}}(t, j)=1, \quad \text { and } \quad \dot{\bar{s}}(t, j)=0,
$$

and for all $t_{j+1}, j \in \mathbb{N}_{0}$, (with $t_{0}=0$ thus excluded)

$$
\bar{\tau}\left(t_{j+1}, j+1\right)=\bar{s}\left(t_{j+1}, j+1\right)=\max \left\{0, \bar{\tau}\left(t_{j+1}, j\right)-\tau_{r-d t}^{*}\right\} .
$$

To complete the proof, we need to show that this hybrid $\operatorname{arc}(\bar{\tau}, \bar{s})$ is a solution to (6). Obviously, the flow and jump dynamics of (6) are satisfied by the defined hybrid arc. As such, it is only required to show for all $j \in \mathbb{N}_{0}$ that

$$
\begin{aligned}
\bar{\tau}\left(t_{j+1}, j\right) & \geq \bar{s}\left(t_{j+1}, j\right)+\tau_{\text {miati }} \\
\bar{\tau}(t, j) & \in\left[0, \tau_{\text {mati }}\right] \text { when } t \in\left[t_{j}, t_{j+1}\right] .
\end{aligned}
$$

For (55a), we first observe that, since $\bar{\tau}\left(t_{1}, 0\right) \geq \bar{\tau}(0,0)=0$ and $\bar{s}\left(t_{1}, 0\right)=\bar{s}(0,0)+\tau_{\text {miati }} \leq 0$ as a result of $(54 \mathrm{a})$, the

\footnotetext{
${ }^{5}$ Note that choosing the initial conditions as such allows for a jump of the hybrid system (6) at any jump time $t_{1} \geq 0$, which is required based on (7).
} 
inequality is satisfied for $j=0$. For all $j \in \mathbb{N}$, we have that $\bar{s}\left(t_{j+1}, j\right)=\bar{s}\left(t_{j}, j\right)=\bar{\tau}\left(t_{j}, j\right)$, as a result of (54b). Moreover, from (7a) it follows that $t_{j+1}-t_{j} \geq \tau_{\text {miati }}, j \in \mathbb{N}$. Recalling now that $\dot{\bar{\tau}}=1$ and $\dot{\bar{s}}=0$, see (54a), the above directly leads for all $j \in \mathbb{N}$ to

$$
\begin{aligned}
\bar{\tau}\left(t_{j+1}, j\right)-\bar{s}\left(t_{j+1}, j\right) & =\bar{\tau}\left(t_{j+1}, j\right)-\bar{s}\left(t_{j}, j\right) \\
& =\bar{\tau}\left(t_{j+1}, j\right)-\bar{\tau}\left(t_{j}, j\right) \\
& =t_{j+1}-t_{j} \geq \tau_{\text {miati }}
\end{aligned}
$$

implying that (55a) indeed holds.

Concerning (55b), obviously $\bar{\tau}(t, j) \geq 0$ for any $(t, j) \in E$ as $\dot{\bar{\tau}}=1$ ( $\bar{\tau}$ is increasing during flows), $\bar{\tau}(0,0) \geq 0$, and $\bar{\tau}$ is not reset to any value lower than 0 . To guarantee also the upper bound $\bar{\tau}(t, j) \leq \tau_{\text {mat }}$, we will proceed by contradiction. That is, we observe that (55b) holds unless there exist hybrid times $(t, j),(\bar{t}, j) \in E$ with $\bar{t}>t$ such that $\bar{\tau}(t, j)=\tau_{\text {mati }}$ and, hence, $\bar{\tau}(\bar{t}, j)>\tau_{\text {mati }} \geq \tau_{r-d t}^{*}$. Suppose that such hybrid times exist and let $(\tilde{t}, k)$ be the largest hybrid time in $E$ smaller than $(t, j)$ (possibly being $(0,0))$ at which $\bar{\tau}(\tilde{t}, k)=0$. Hence, we have that any jump that occurs after the point $(\tilde{t}, k)$ always leads to a full decrease of $\tau_{r-d t}^{*}$ time units for the value of $\tau$. As such, the above implies that

$$
\begin{aligned}
\tau_{\text {mati }}=\bar{\tau}(t, j) & =\bar{\tau}(\tilde{t}, k)+(t-\tilde{t})-(j-k) \tau_{r-d t}^{*} \\
& =(t-\tilde{t})-(j-k) \tau_{r-d t}^{*} \\
& <(\bar{t}-\tilde{t})-(j-k) \tau_{r-d t}^{*} \\
\Rightarrow j-k & <\frac{(\bar{t}-\tilde{t})-\tau_{m a t i}}{\tau_{r-d t}^{*}},
\end{aligned}
$$

which contradicts that $E$ satisfies (7b). Hence, also (55b) must hold. This completes the proof.

Proof of Lemma 1: Consider the function $\phi_{W}^{*}$ : $\left[0, \tau_{\text {mati }}\right] \rightarrow \mathbb{R}_{\geq 0}$ that is for all $\tau \in\left[0, \tau_{\text {mati }}\right]$ given by the solution to

$$
\frac{\mathrm{d}}{\mathrm{d} \tau} \phi_{W}^{*}(\tau)=\lambda_{W} \phi_{W}^{*}(\tau)
$$

with $\lambda_{W}$ given by (31) such that

$$
\begin{aligned}
\phi_{W}^{*}(\tau) & =e^{\lambda_{W} \tau} \phi_{W}^{*}(0) \\
& =e^{-\left(\varepsilon^{2}-\delta\right) \tau_{\text {miati }}} \frac{\tau}{\tau_{r-d t}^{*}}\left(\lambda^{2}\right)^{\frac{\tau}{\tau_{r-d t}^{*}}} \phi_{W}^{*}(0) .
\end{aligned}
$$

Observe that, since $\ln \left(\lambda^{2}\right)<0$ (as $\lambda<1$ ) and $\delta \leq \varepsilon^{2}, \phi_{W}^{*}$ is a strictly decreasing function and, hence, it holds for any $\tau_{1}, \tau_{2} \in\left[0, \tau_{\text {mati }}\right]$ such that $\tau_{2}-\tau_{1} \leq \tau_{r-d t}^{*}$ that

$$
\begin{aligned}
\frac{\phi_{W}^{*}\left(\tau_{1}\right)}{\phi_{W}^{*}\left(\tau_{2}\right)} & =e^{-\left(\varepsilon^{2}-\delta\right) \tau_{\text {miati }} \frac{\left(\tau_{1}-\tau_{2}\right)}{\tau_{r-d t}^{*}}}\left(\lambda^{2}\right)^{\frac{\left(\tau_{1}-\tau_{2}\right)}{\tau_{r-d t}^{*}}} \\
& \leq e^{-\left(\varepsilon^{2}-\delta\right) \tau_{\text {miati }}} \frac{-\tau_{r-d t}^{*}}{\tau_{r-d t}^{*}}\left(\lambda^{2}\right)^{\frac{-\tau_{r-d t}^{*}}{\tau_{r-d t}^{*}}} \\
& =e^{\left(\varepsilon^{2}-\delta\right) \tau_{\text {miati }}} \frac{1}{\lambda^{2}},
\end{aligned}
$$

Taking now $\phi_{W}^{*}(0)=\tilde{\phi}_{W}(0)$, it follows from (30) and the comparison lemma [38, Lemma 3.4] that

$$
\tilde{\phi}_{W}(\tau) \geq \phi_{W}^{*}(\tau) \text { for all } \tau \in\left[0, \tau_{\text {mati }}\right] .
$$

Moreover, we have for any $\tau_{1}, \tau_{2} \in\left[0, \tau_{\text {mati }}\right], \tau_{2} \geq \tau_{1}$, that (30) in combination with $\phi_{W}^{*}$ as defined in (56) also implies

$$
\begin{aligned}
\tilde{\phi}_{W}\left(\tau_{2}\right)-\tilde{\phi}_{W}\left(\tau_{1}\right) & =\int_{\tau_{1}}^{\tau_{2}} \frac{\mathrm{d}}{\mathrm{d} s} \tilde{\phi}_{W}(s) \mathrm{d} s \\
& \geq \int_{\tau_{1}}^{\tau_{2}} \frac{\mathrm{d}}{\mathrm{d} s} \phi_{W}^{*}(s) \mathrm{d} s=\phi_{W}^{*}\left(\tau_{2}\right)-\phi_{W}^{*}\left(\tau_{1}\right) .
\end{aligned}
$$

Finally, we can rewrite (57) as

$$
\phi_{W}^{*}\left(\tau_{2}\right) \geq e^{-\left(\varepsilon^{2}-\delta\right) \tau_{\text {miati }}} \lambda^{2} \phi_{W}^{*}\left(\tau_{1}\right)
$$

or, alternatively, as

$$
\phi_{W}^{*}\left(\tau_{2}\right)-\phi_{W}^{*}\left(\tau_{1}\right) \geq \underbrace{\left(e^{-\left(\varepsilon^{2}-\delta\right) \tau_{\text {miati }}} \lambda^{2}-1\right)}_{<0 \text { since } \lambda<1 \text { and } \delta \leq \varepsilon^{2}, \tau_{\text {miat } i}>0} \phi_{W}^{*}\left(\tau_{1}\right)
$$

for any $\tau_{1}, \tau_{2} \in\left[0, \tau_{\text {mati }}\right]$ such that $\tau_{2}-\tau_{1} \leq \tau_{r-d t}^{*}$.

Combining now all of the above, we obtain for any $\tau_{1}, \tau_{2} \in$ $\left[0, \tau_{\text {mati }}\right]$ such that $\tau_{2}-\tau_{1} \leq \tau_{r-d t}^{*}$ that

$$
\begin{aligned}
\tilde{\phi}_{W}\left(\tau_{2}\right)-\tilde{\phi}_{W}\left(\tau_{1}\right) & \stackrel{(59)}{\geq} \phi_{W}^{*}\left(\tau_{2}\right)-\phi_{W}^{*}\left(\tau_{1}\right) \\
& \stackrel{(60)}{\geq}\left(e^{-\left(\varepsilon^{2}-\delta\right) \tau_{m i a t i}} \lambda^{2}-1\right) \phi_{W}^{*}\left(\tau_{1}\right) \\
& \stackrel{(58)}{\geq}\left(e^{-\left(\varepsilon^{2}-\delta\right) \tau_{\text {miati }}} \lambda^{2}-1\right) \tilde{\phi}_{W}\left(\tau_{1}\right) \\
\Rightarrow \quad \tilde{\phi}_{W}\left(\tau_{2}\right) & \geq\left(e^{-\left(\varepsilon^{2}-\delta\right) \tau_{\text {miati }}} \lambda^{2}-1\right) \tilde{\phi}_{W}\left(\tau_{1}\right)+\tilde{\phi}_{W}\left(\tau_{1}\right) \\
& =e^{-\left(\varepsilon^{2}-\delta\right) \tau_{\text {miati }}} \lambda^{2} \tilde{\phi}_{W}\left(\tau_{1}\right),
\end{aligned}
$$

which directly gives us condition (29).

\section{APPENDIX B}

\section{NOTE ON THE OPTIMALITY OF [12]}

As already mentioned in Section IV-E, the value for the MATI $T^{[12]}$ is computed in [12] as the point in time for which the function $\phi:\left[0, T^{[12]}\right] \rightarrow \mathbb{R}$ given by the nonlinear differential equation

$$
\frac{\mathrm{d}}{\mathrm{d} \tau} \phi(\tau)=-2 L-\gamma\left(\phi^{2}(\tau)-1\right) .
$$

reaches the value $\lambda^{2} \phi(0)$ for some $\lambda \in(0,1)$, which follows from the used scheduling protocol. As such, the value for the initial condition $\phi(0)$ influences the value for the MATI, and, hence, can be optimized. Therefore, in this note, the value for the MATI is first computed as a function of the initial condition. That is, by definition of (61) with $\phi(0)=\phi_{0}$ and $\phi_{W}\left(T^{[12]}\right)=\lambda^{2} \phi_{0}$, we can write, see also the proof of $[12$, Lemma 2],

$$
T^{[12]}\left(\phi_{0}\right)=-\int_{\phi_{0}}^{\lambda^{2} \phi_{0}}\left(\frac{1}{\gamma \phi^{2}+2 L \phi+\gamma}\right) \mathrm{d} \phi .
$$

The goal is now to obtain the 'optimal' value for $\phi_{0}$ such that the value for the MATI is as high as possible. To this end, define $s:=\phi+\frac{L}{\gamma}$ and $r:=\sqrt{\left|\left(\frac{\gamma}{L}\right)^{2}-1\right|}$ and observe that

$$
s^{2}-\operatorname{sign}\left(L^{2}-\gamma^{2}\right)\left(\frac{L r}{\gamma}\right)^{2}=\frac{1}{\gamma}\left(\gamma \phi^{2}+2 L \phi+\gamma\right),
$$


where $\operatorname{sign}(\cdot)$ is the sign function with $\operatorname{sign}(0)=0$. Hence, we can rewrite (62) using the transformation defined by $s$ as

$$
\begin{aligned}
T^{[12]}\left(\phi_{0}\right) & =-\frac{1}{\gamma} \int_{\underline{s}}^{\bar{s}}\left(s^{2}-\operatorname{sign}\left(L^{2}-\gamma^{2}\right)\left(\frac{L r}{\gamma}\right)^{2}\right)^{-1} \frac{\mathrm{d} \phi}{\mathrm{d} s} \mathrm{~d} s \\
& =-\frac{1}{\gamma} \int_{\underline{s}}^{\bar{s}}\left(s^{2}-\operatorname{sign}\left(L^{2}-\gamma^{2}\right)\left(\frac{L r}{\gamma}\right)^{2}\right)^{-1} \mathrm{~d} s
\end{aligned}
$$

with $\bar{s}:=\lambda^{2} \phi_{0}+\frac{L}{\gamma}$ and $\underline{s}:=\phi_{0}+\frac{L}{\gamma}$. The value for the MATI is now obtained by analyzing the various scenarios:

- In the first scenario where $\gamma>L$, we use the fact that

$$
\begin{aligned}
-\frac{1}{\gamma} \int_{\underline{s}}^{\bar{s}}\left(s^{2}+\right. & \left.\left(\frac{L r}{\gamma}\right)^{2}\right)^{-1} \mathrm{~d} s \\
& =-\frac{1}{L r}\left(\arctan \left(\frac{\bar{s} \gamma}{L r}\right)-\arctan \left(\frac{\underline{s} \gamma}{L r}\right)\right)
\end{aligned}
$$

and that for all $c_{2} \geq c_{1} \geq 0$ we have that

$$
\arctan \left(c_{2}\right)-\arctan \left(c_{1}\right)=\arctan \left(\frac{c_{2}-c_{1}}{1+c_{1} c_{2}}\right),
$$

resulting in

$$
T^{[12]}\left(\phi_{0}\right)=\frac{1}{L r} \arctan \left(\frac{L r \gamma(\underline{s}-\bar{s})}{L^{2} r^{2}+\bar{s} \underline{s} \gamma^{2}}\right) .
$$

Using now the upper and lower bounds of the integration we obtain that

$$
\operatorname{Lr} \gamma(\underline{s}-\bar{s})=\operatorname{Lr} \gamma \phi_{0}\left(1-\lambda^{2}\right)=\operatorname{Lr} \gamma \phi_{0}(1-\lambda)(1+\lambda)
$$

and that, by using $L^{2} r^{2}=\gamma^{2}-L^{2}$,

$$
\begin{aligned}
L^{2} r^{2}+\bar{s} \underline{s} \gamma^{2} & =L^{2} r^{2}+\gamma^{2}\left(\lambda^{2} \phi_{0}+\frac{L}{\gamma}\right)\left(\phi_{0}+\frac{L}{\gamma}\right) \\
& =\left(\gamma^{2}-L^{2}\right)+\gamma^{2}\left(\lambda^{2} \phi_{0}^{2}+\frac{L}{\gamma} \phi_{0}\left(1+\lambda^{2}\right)+\frac{L^{2}}{\gamma^{2}}\right) \\
& =\gamma^{2}+\gamma^{2} \lambda^{2} \phi_{0}^{2}+L \gamma \phi_{0}\left(1+\lambda^{2}\right),
\end{aligned}
$$

which directly yields

$$
T^{[12]}\left(\phi_{0}\right)=\frac{1}{L r} \arctan \left(\frac{L\left(1-\lambda^{2}\right) \phi_{0} r}{L\left(1+\lambda^{2}\right) \phi_{0}+\gamma \lambda^{2} \phi_{0}^{2}+\gamma}\right)
$$

with $\frac{\partial T^{[12]}\left(\phi_{0}\right)}{\partial \phi_{0}}=\gamma\left(\frac{1}{\left(L+\gamma \phi_{0}\right)^{2}+L^{2} r^{2}}-\frac{\lambda^{2}}{\left(L+\gamma \lambda^{2} \phi_{0}\right)^{2}+L^{2} r^{2}}\right)$. To obtain now the value for $\phi_{0}$, which maximizes the value for the MATI, we compute the zeros of the above partial derivative with respect to $\phi_{0}$. This results in

$$
\begin{array}{rrrr} 
& & \frac{1}{\gamma+2 L \phi_{0}+\gamma \phi_{0}^{2}}-\frac{\lambda^{2}}{\gamma+2 L \lambda^{2} \phi_{0}+\gamma \lambda^{4} \phi_{0}^{2}} & =0 \\
\lambda^{2} \gamma+\gamma \lambda^{2} \phi_{0}^{2}-\gamma-\gamma \phi_{0}^{2} \lambda^{4} & =0 \\
\Rightarrow & & \phi_{0}=\frac{1}{\lambda} \text { or } \phi_{0}=-\frac{1}{\lambda} .
\end{array}
$$

As $\phi_{0}$ should be positive, we disregard the second solution (implying that there is only a single solution $\phi_{0}$ for which $\left.\frac{\partial T^{12]}\left(\phi_{0}\right)}{\partial \phi_{0}}=0\right)$ and by verifying that

$$
\frac{\partial^{2} T^{[12]}\left(\lambda^{-1}\right)}{\partial \phi_{0}^{2}}=\frac{2 \gamma \lambda^{3}\left(\lambda^{2}-1\right)}{\left(\gamma+2 L \lambda+\gamma \lambda^{2}\right)^{2}}<0
$$

we can conclude that having $\phi_{0}=\lambda^{-1}$ results in the global maximal value for the MATI $T^{[12]}$.
- In the second scenario where $\gamma=L$, it follows that

$$
\begin{aligned}
T^{[12]}\left(\phi_{0}\right) & =-\frac{1}{\gamma} \int_{\underline{s}}^{\bar{s}} \frac{1}{s^{2}} \mathrm{~d} s=\frac{1}{\gamma}\left(\frac{1}{\bar{s}}-\frac{1}{\underline{s}}\right) \\
& =\frac{1}{\gamma}\left(\frac{1}{\lambda^{2} \phi_{0}+1}-\frac{1}{\phi_{0}+\lambda}\right) .
\end{aligned}
$$

Computing now again the zeros of the partial derivative with respect to $\phi_{0}$ by means of

$$
\begin{aligned}
& \frac{\partial T^{[12]}\left(\phi_{0}\right)}{\partial \phi_{0}}=\frac{1}{\gamma^{2}}\left(\frac{1}{\left(1+\phi_{0}\right)^{2}}-\frac{\lambda^{2}}{\left(1+\lambda^{2} \phi_{0}\right)^{2}}\right)=0 \\
& \Rightarrow \quad\left(1+\lambda^{2} \phi_{0}\right)^{2}-\lambda^{2}\left(1+\phi_{0}\right)^{2}=0 \\
& \Rightarrow \quad \lambda^{2}\left(\lambda^{2}-1\right) \phi_{0}^{2}-\left(\lambda^{2}-1\right)=0 \\
& \Rightarrow \quad \phi_{0}=\frac{1}{\lambda} \text { or } \phi_{0}=-\frac{1}{\lambda} \text {, }
\end{aligned}
$$

and verifying that

$$
\frac{\partial^{2} T^{[12]}\left(\lambda^{-1}\right)}{\partial \phi_{0}^{2}}=\frac{2(\lambda-1) \lambda^{3}}{\gamma\left(1+\lambda^{3}\right)}<0,
$$

we obtain the same conclusion as in the first item that $\phi_{0}=\lambda^{-1}$ results in the highest possible value for $T^{[12]}$.

- For the third scenario when $\gamma<L$, we directly obtain (following the same analysis as in the first item, see also the proof of [12, Lemma 2]) that

$$
T^{[12]}\left(\phi_{0}\right)=\frac{1}{L r} \operatorname{arctanh}\left(\frac{L\left(1-\lambda^{2}\right) \phi_{0} r}{L\left(1+\lambda^{2}\right) \phi_{0}+\gamma \lambda^{2} \phi_{0}^{2}+\gamma}\right),
$$

which has a partial derivative with respect to $\phi_{0}$ equal to the one from the first item, resulting in the same conclusion that having $\phi_{0}=\lambda^{-1}$ results in the highest possible value for $T^{[12]}$.

\section{ACKNOWLEDGMENT}

The authors want to thank D. Carnevale and A.R. Teel for their insightful discussions and suggestions on Appendix B.

\section{REFERENCES}

[1] W. Zhang, M.S. Branicky, and S.M. Phillips, "Stability of networked control systems," IEEE Control Systems, vol. 21, no. 1, pp. 84-99, 2001.

[2] Y. Tipsuwan and M.Y. Chow, "Control methodologies in networked control systems," Control engineering practice, vol. 11, no. 10, pp. 1099-1111, 2003.

[3] T.C. Yang, "Networked control system: A brief survey," IEE Proceedings Control Theory and Applications, vol. 153, no. 4, p. 403, 2006.

[4] J.P. Hespanha, P. Naghshtabrizi, and Y. Xu, "A survey of recent results in networked control systems," Proceedings of the IEEE, vol. 95, no. 1, pp. 138-162, 2007.

[5] H. Gao, T. Chen, and J. Lam, "A new delay system approach to networkbased control," Automatica, vol. 44, no. 1, pp. 39-52, 2008.

[6] L. Hetel, C. Fiter, H. Omran, A. Seuret, E. Fridman, J. Richard, and S.I. Niculescu, "Recent developments on the stability of systems with aperiodic sampling: An overview," Automatica, vol. 76, pp. 309-335, 2017.

[7] M.B.G. Cloosterman, N. Van de Wouw, W.P.M.H. Heemels, and H. Nijmeijer, "Stability of networked control systems with uncertain timevarying delays," IEEE Transactions on Automatic Control, vol. 54, no. 7, pp. 1575-1580, 2009.

[8] G.C. Walsh, O. Beldiman, and L.G. Bushnell, "Asymptotic behavior of nonlinear networked control systems," IEEE Transactions on Automatic Control, vol. 46, no. 7, pp. 1093-1097, 2001.

[9] G.C. Walsh, H. Ye, and L.G. Bushnell, "Stability analysis of networked control systems," IEEE Transactions on Control Systems Technology, vol. 10, no. 3, pp. 438-446, 2002. 
[10] D. Nešić and A.R. Teel, "Input-to-state stability of networked control systems," Automatica, vol. 40, no. 12, pp. 2121-2128, 2004.

[11] D. Nešić and A.R. Teel, "Input-output stability properties of networked control systems," IEEE Transactions on Automatic Control, vol. 49, no. 10, pp. 1650-1667, 2004.

[12] D. Carnevale, A.R. Teel, and D. Nešić, "A Lyapunov proof of an improved maximum allowable transfer interval for networked control systems," IEEE Transactions on Automatic Control, vol. 52, no. 5, pp. 892-897, 2007.

[13] D. Nešić, A.R. Teel, and D. Carnevale, "Explicit computation of the sampling period in emulation of controllers for nonlinear sampled-data systems," IEEE Transactions on Automatic Control, vol. 54, no. 3, pp. 619-624, 2009.

[14] M. Tabbara, D. Nešić, and A.R. Teel, "Stability of wireless and wireline networked control systems," IEEE Transactions on Automatic Control, vol. 52, no. 9, pp. 1615-1630, 2007.

[15] W.P.M.H. Heemels, A.T. Teel, N. van de Wouw, and D. Nešić, "Networked control systems with communication constraints: Tradeoffs between transmission intervals, delays, and performance," IEEE Transactions on Automatic Control, vol. 55, no. 8, pp. 1781-1796, 2010.

[16] T. Ahmed-Ali and F. Lamnabhi-Lagarrigue, "High gain observer design for some networked control systems," IEEE Transactions on Automatic Control, vol. 57, no. 4, pp. 995-1000, 2012.

[17] W.P.M.H. Heemels, D.P. Borgers, N. van de Wouw, D. Nešić, and A.R. Teel, "Stability analysis of nonlinear networked control systems with asynchronous communication: A small-gain approach," in Proceedings of the IEEE $52^{\text {th }}$ Conference on Decision and Control, 2013, pp. 46314637.

[18] R. Postoyan, N. Van de Wouw, D. Nešić, and W.P.M.H. Heemels, "Tracking control for nonlinear networked control systems," IEEE Transactions on Automatic Control, vol. 59, no. 6, pp. 1539-1554, 2014.

[19] L.H. Duc, D. Christmann, R. Gotzhein, S. Siegmund, and F. Wirth, "The stability of try-once-discard for stochastic communication channels: Theory and validation," in Proceedings of the IEEE $54^{\text {th }}$ Annual Conference on Decision and Control (CDC'15), 2015, pp. 4170-4175.

[20] K.Z. Liu, R. Wang, and G.P. Liu, "Tradeoffs between transmission intervals and delays for decentralized networked control systems based on a gain assignment approach," IEEE Transactions on Circuits and Systems II: Express Briefs, vol. 63, no. 5, pp. 498-502, 2016.

[21] S.H.J. Heijmans, D.P. Borgers, and W.P.M.H. Heemels, "Stability and performance analysis of spatially invariant systems with networked communication," IEEE Transactions on Automatic Control, vol. 62 , no. 10, pp. 4994-5009, 2017.

[22] S.H.J. Heijmans, R. Postoyan, D. Nešić, and W.P.M.H. Heemels, "Computing minimal and maximal allowable transmission intervals for networked control systems using the hybrid systems approach," IEEE Control Systems Letters, vol. 1, no. 1, pp. 56-61, 2017.

[23] S.H.J. Heijmans, D. Nešić, R. Postoyan, and W.P.M.H. Heemels, "A generalized hybrid Lyapunov proof for networked control systems: Improving the maximum allowable transmission interval," in Proceedings of the IEEE 57th Conference on Decision and Control, 2018, pp. 428433.

[24] S. Adyanthaya, Z. Zhang, M. Geilen, J. Voeten, T. Basten, and R. Schiffelers, "Robustness analysis of multiprocessor schedules," in Proceedings of the International Conference on the Embedded Computer Systems: Architectures, Modeling, and Simulation (SAMOS XIV), 2014, pp. 9-17.

[25] J.P. Hespanha, D. Liberzon, and A.R. Teel, "On input-to-state stability of impulsive systems," in Proceedings of the IEEE $44^{\text {th }}$ Conference on Decision and Control, 2005, pp. 3992-3997.

[26] — - "Lyapunov conditions for input-to-state stability of impulsive systems," Automatica, vol. 44, no. 11, pp. 2735-2744, 2008.

[27] S.H.J. Heijmans, R. Postoyan, D. Nešić, and W.P.M.H. Heemels, "Reverse average dwell-times for networked control systems," in Proceedings of the IEEE $58^{\text {th }}$ Annual Conference on Decision and Control (CDC'19), 2019, submitted for publication.

[28] R. Goebel, R.G. Sanfelice, and A.R. Teel, Hybrid dynamical systems. United Kingdom: Princeton University Press, 2012.

[29] D. Nešić and A.R. Teel, "A Lyapunov-based small-gain theorem for hybrid ISS systems," in Proceedings of the $47^{\text {th }}$ IEEE Conference on Decision and Control, 2008, pp. 3380-3385.

[30] D. Nešić, A.R. Teel, G. Valmorbida, and L. Zaccarian, "Finite-gain $\mathcal{L}_{p^{-}}$ stability for hybrid dynamical systems," Automatica, vol. 49, no. 8, pp. 2384-2396, 2013.

[31] C. Cai, A.R. Teel, and R. Goebel, "Smooth Lyapunov functions for hybrid systems part II: (pre-)Asymptotically stable compact sets," IEEE Transactions on Automatic Control, vol. 53, no. 3, pp. 734-748, 2008.
[32] A.N. Michel and L. Hou, "Relaxation of hypotheses in LaSalleKrasovskii-type invariance results," SIAM Journal on Control and Optimization, vol. 49, no. 4, pp. 1383-1403, 2011

[33] A.R. Teel, F. Forni, and L. Zaccarian, "Lyapunov-based sufficient conditions for exponential stability in hybrid systems," IEEE Transactions on Automatic Control, vol. 58, no. 6, pp. 1591-1596, 2013.

[34] A.A. Ahmadi and P.A. Parrilo, "Non-monotonic Lyapunov functions for stability of discrete time nonlinear and switched systems," in Proceedings of the $47^{\text {th }}$ IEEE Conference on Decision and Control (CDC'08), 2008, pp. 614-621.

[35] M. Chini, "Sull'integrazione di alcune equazioni differenziali del primo ordine," Rendiconti Instituto Lombardo (2), vol. 57, pp. 506-511, 1924.

[36] M. Chamberland and A. Gasull, "Chini equations and isochronous centers in three-dimensional differential systems," Qualitative Theory of Dynamical Systems, vol. 9, no. 1-2, pp. 29-38, 2010.

[37] E. Kamke, Differentialgleichungen: Losungsmethoden und losungen, I, Gewohnliche differentialgleichungen. Leipzig: B.G. Teubner, 1977.

[38] H.K. Khalil, Nonlinear systems, 3rd ed. Upper Saddle River, New Jersey: Prentice-Hall, Inc., 2002.

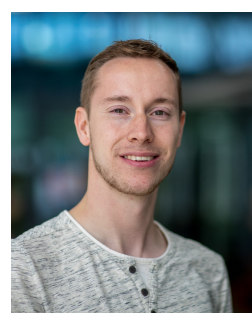

S.H.J. (Stefan) Heijmans received the M.Sc. degree (Cum Laude) and Ph.D. degree both in Mechanical Engineering from the Eindhoven University of Technology (TU/e), Eindhoven, the Netherlands, in 2015 and 2019, respectively. Moreover, his Ph.D. research was awarded with the DISC PhD Award 2019. Since September 2019, he has been working at Demcon Advanced Mechatronics B.V. as a software engineer, developing his competence in embedded software and machine learning.

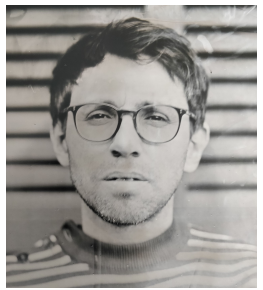

R. (Romain) Postoyan received the M.Sc. degree in Electrical and Control Engineering from ENSEEIHT (France) in 2005, the M.Sc. by Research in Control Theory \& Application from Coventry University (United Kingdom) in 2006, and the Ph.D. in Control Theory from Université Paris-Sud (France) in 2009. Since 2011, he is a CNRS researcher at the "Centre de Recherche en Automatique de Nancy" (France). He received the "Habilitation à Diriger des Recherches (HDR)" in 2019 from Université de Lorraine (Nancy, France).

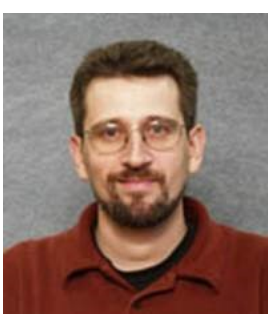

D. (Dragan) Nešić (F'08) received the Ph.D. degree in systems engineering from the Research School of Information Sciences, Australian National University, Canberra, ACT, Australia, in 1997. Since 1999, he has been with The University of Melbourne, Melbourne, VIC, Australia, where he is currently a Professor with the Department of Electrical and Electronic Engineering

Dr. Nešić served as a member for the Board of Governors, CSS, and as a General Co-Chair of the IEEE CDC 2017. He was a co-recipient of the George S. Axelby Outstanding Paper Award in 2018. He was a recipient of Humboldt Research Fellowship in 2003 by the Alexander von Humboldt Foundation, an Australian Professorial Fellowship from 2004 to 2009, and Future Fellowship from 2010 to 2014 by the Australian Research Council. $\mathrm{He}$ also served as an Associate Editor for the journals Automatica, the IEEE Transactions on Automatic Control, Systems and Control Letters, European Journal of Control.

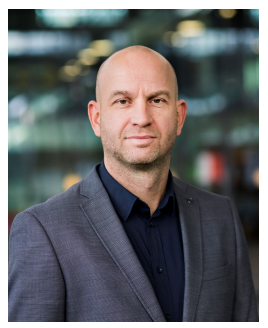

W.P.M.H. (Maurice) Heemels (M'07-SM'10-F'16) received the M.Sc. degree in mathematics and the $\mathrm{Ph} . \mathrm{D}$. degree in control theory (both cum laude) from the Eindhoven University of Technology (TU/e), the Netherlands, in 1995 and 1999, respectively. From 2000 to 2004, he was with the Electrical Engineering Department, TU/e and from 2004 to 2006 with the Embedded Systems Institute (ESI). Since 2006, he has been with the Department of Mechanical Engineering, TU/e, where he is currently a Full Professor. His current research interests include general system and control theory, hybrid and cyber-physical systems, networked and event-triggered control systems and constrained systems including model predictive control. Dr. Heemels served/s on the editorial boards of Automatica, Nonlinear Analysis: Hybrid Systems, Annual Reviews in Control, and IEEE Transactions on Automatic Control. He was a recipient of a personal VICI grant awarded by NWO. 